\title{
ABSOLUTE AND RELATIVE GROMOV-WITTEN INVARIANTS OF VERY AMPLE HYPERSURFACES
}

\author{
ANDREAS GATHMANN
}

\begin{abstract}
For any smooth complex projective variety $X$ and smooth very ample hypersurface $Y \subset X$, we develop the technique of genus zero relative Gromov-Witten invariants of $Y$ in $X$ in algebro-geometric terms. We prove an equality of cycles in the Chow groups of the moduli spaces of relative stable maps that relates these relative invariants to the Gromov-Witten invariants of $X$ and $Y$. Given the Gromov-Witten invariants of $X$, we show that these relations are sufficient to compute all relative invariants, as well as all genus zero Gromov-Witten invariants of $Y$ whose homology and cohomology classes are induced by $X$.
\end{abstract}

Much work has been done recently on Gromov-Witten invariants related to hypersurfaces. There are essentially two different problems that have been studied. The first one is the question: how can one compute the Gromov-Witten invariants of a hypersurface from those of the ambient variety $[\mathrm{Be}],[\mathrm{G}],[\mathrm{K}],[\mathrm{LLY}]$ ? The second problem, mainly studied from the point of view of symplectic geometry, is the theory of relative Gromov-Witten invariants of a hypersurface [IP1], [IP2], [LR], [/R], [V]. The goal of this paper is to show that these two problems that have been studied completely independently so far are in fact very closely related.

Let $X$ be a smooth complex projective variety and $Y \subset X$ a smooth very ample hypersurface. We start by giving a very short description of our method to compute the genus zero Gromov-Witten invariants of $Y$ in terms of those of $X$, skipping all technical details.

Fix $n \geq 1$ and $\beta \in H_{2}(X)$. For $m \geq 0$, we let $\bar{M}_{(m)}$ (the official notation will be $\left.\bar{M}_{(m, 0, \ldots, 0)}(X, \beta)\right)$ be a suitable compactification of the moduli space of all irreducible stable maps $\left(\mathbb{P}^{1}, x_{1}, \ldots, x_{n}, f\right)$ to $X$ such that $f$ has multiplicity at least $m$ to $Y$ at the point $x_{1}$. Obviously, $\bar{M}_{(0)}$ should be just the ordinary moduli space of stable maps to $X$. On the other hand, $\bar{M}_{(Y \cdot \beta+1)}$ should correspond to the moduli space of stable maps to $Y$, as all irreducible curves in $X$ having multiplicity $Y \cdot \beta+1$ to $Y$ must actually lie inside $Y$. Moreover, $\bar{M}_{(m+1)}$ is a subspace of $\bar{M}_{(m)}$ of (expected) codimension one.

The strategy is now obvious: if we can describe the (virtual) divisor $\bar{M}_{(m+1)}$ in $\bar{M}_{(m)}$ intersection-theoretically in terms of known classes (and our main theorem 2.6 does precisely that), then we can compute intersection products on $\bar{M}_{(m+1)}$ if we can compute them on $\bar{M}_{(m)}$. Iterating this procedure for $m$ from 0 to $Y \cdot \beta$, this means that we can compute the Gromov-Witten invariants of $Y$ if we can compute the Gromov-Witten invariants of $X$. In fact, we will show in a forthcoming paper that this method reproves and generalizes the well-known "mirror symmetry" type formulas for Gromov-Witten invariants of certain hypersurfaces [B], $[\mathrm{G},[\mathrm{LLY}]$.

Funded by the DFG scholarship Ga 636/1-1. 
Let us make the step from multiplicity $m$ to $m+1$ a bit more precise. It is easily seen that there is a section of a line bundle $L_{(m)}$ on $\bar{M}_{(m)}$ whose zero locus describes exactly the condition that $f$ vanishes to order at least $m+1$ along $Y$ at $x_{1}$. Hence one would naïvely expect that $\bar{M}_{(m+1)}$ is just the first Chern class of $L_{(m)}$, which turns out to be $m \psi+e v^{*} Y$ (where $\psi$ is the cotangent line class and $e v$ the evaluation map at the first marked point). However, this intuition breaks down for those stable maps where $x_{1}$ lies on a component that is completely mapped to $Y$ by $f$ (see the picture in construction 2.1), as $f$ actually has infinite multiplicity to $Y$ at $x_{1}$ in this case. Thus we get correction terms from reducible curves of that kind in our final equation. These correction terms are quite complicated, but they can be recursively computed as they are made up of invariants of smaller degree.

In this paper we will define more general spaces than the $\bar{M}_{(m)}$ mentioned above. Namely, we allow the specification of multiplicities to $Y$ not only at the point $x_{1}$ but at all marked points. We call those moduli spaces the spaces of relative stable maps, and equip them with virtual fundamental classes. Intersection products on them are then called relative Gromov-Witten invariants. Of course, they have the obvious (possibly virtual) geometric interpretation as numbers of curves having given multiplicities to $Y$ and satisfying some additional incidence conditions.

It should be said clearly that the specification of more than one multiplicity is not necessary if one only wants to compute the Gromov-Witten invariants of $Y$ from those of $X$. However, the general case fits nicely into the picture and establishes the connection to the existing literature on relative Gromov-Witten invariants, as these invariants have only been considered so far in the case where the sum of the multiplicities is equal to $Y \cdot \beta$ (i.e. where "all intersection points with $Y$ are marked").

The outline of the paper is as follows. In section 1 we define the moduli spaces of relative stable maps and define their virtual fundamental classes. The construction of the line bundles $L_{(m)}$ and the moduli spaces for the correction terms mentioned above is given in section 2. At the end of this section we state our main theorem 2.6 that describes how the moduli spaces of relative invariants change if one of the multiplicities is increased by one. The proof of this theorem is done in two steps. In the first step in section 3 we look at the special case where $Y \subset X$ is a hyperplane in projective space. In this case no virtual fundamental classes are needed, and the main theorem is established by purely geometric analysis. The ideas for the main proofs of this section are taken from $\| \mathrm{V} \mid$. In the second step in section A, we prove the general case by "pulling back" the result for hyperplanes in $\mathbb{P}^{N}$ along the morphism $\bar{M}_{n}(X, \beta) \rightarrow \bar{M}_{n}\left(\mathbb{P}^{N}, d\right)$ induced by the complete linear system $|Y|$. Finally, in section 5 we prove that the main theorem can be used to compute the absolute and relative Gromov-Witten invariants of $Y$ in terms of the Gromov-Witten invariants of $X$. In a forthcoming paper, we will study the structure of these computations and give some explicit examples.

A few remarks seem in order how this work is related to the existing literature. The original ideas and motivation for our paper come from the work of R. Vakil [V], who proved the main theorem under the following restrictions: $Y \subset X$ is a hyperplane in $\mathbb{P}^{N}$, the sum of the prescribed multiplicities is equal to the degree of the curves, and one of the multiplicities is raised from zero to one. It is interesting to note that he used the main theorem in the opposite direction, namely to compute the invariants of $X$ from those of $Y$. But the algorithm used there is very specific to the case of a hyperplane in $\mathbb{P}^{N}$; it does not work for general $Y \subset X$. 
All methods that have been known so far to compute Gromov-Witten invariants of hypersurfaces $Y \subset X$ need the existence of a torus action on $X$ and use the techniques of equivariant cohomology and fixed point localization. In the case where $Y$ is Calabi-Yau or Fano, the "mirror symmetry" results of A. Givental [G] and B. Lian et al. [LLY] relate the Gromov-Witten invariants of $Y$ to those of $X$ and express them in terms of certain hypergeometric functions. Our methods are completely different; they do not place any restrictions on the variety $X$ and do not require $Y$ to be Calabi-Yau or Fano. In a forthcoming paper we will show that our equations actually lead to the same hypergeometric functions as mentioned above.

Recently A. Bertram $\sqrt{\mathrm{Be}}]$ has found another way to compute certain GromovWitten invariants of Calabi-Yau and Fano hypersurfaces in projective space. He also uses the torus action method, but does the actual computations in a different way. It seems that his computations are closely related to ours, but the exact relation to our methods is still unclear.

Relative Gromov-Witten invariants of any genus have been introduced in symplectic geometry by A. Li and Y. Ruan LR as well as E. Ionel and T. Parker IIP1, IIP2. They have been defined for any codimension two symplectic submanifold $Y$ of a symplectic manifold $X$. The main application in symplectic geometry is the splitting formula that expresses the Gromov-Witten invariants of a symplectic sum $X_{1} \#_{Y} X_{2}$ in terms of the relative Gromov-Witten invariants of $Y$ in $X_{1}$ and $X_{2}$. E. Ionel has informed me that [IP2] together with the results announced in [IP1] can be used to prove a statement in the symplectic category that is analogous to our main theorem.

The author would like to thank T. Graber, J. Harris, and R. Vakil for numerous discussions. This work has been done at the Harvard University, to which the author is grateful for hospitality.

\section{Moduli SPaces of Relative Stable maps}

We begin with the description of the set-up and the definition of the moduli spaces of relative stable maps. Let $X$ be a smooth complex projective variety and $Y \subset X$ a smooth very ample hypersurface. For notational convenience, we denote by $A^{*}(X)$ the ring of algebraic cohomology classes of $X$ modulo torsion, and by $H_{2}^{+}(X)$ the group of effective algebraic homology classes of dimension two, modulo torsion.

Let $\alpha=\left(\alpha_{1}, \ldots, \alpha_{n}\right)$ be an $n$-tuple of non-negative integers. As usual, for such an $n$-tuple we define $|\alpha|:=n$ and $\sum \alpha:=\sum_{i=1}^{n} \alpha_{i}$. If $\alpha=\left(\alpha_{1}, \ldots, \alpha_{n}\right)$ and $\alpha^{\prime}=\left(\alpha_{1}^{\prime}, \ldots, \alpha_{m}^{\prime}\right)$, we write $\alpha \cup \alpha^{\prime}$ for $\left(\alpha_{1}, \ldots, \alpha_{n}, \alpha_{1}^{\prime}, \ldots, \alpha_{m}^{\prime}\right)$. For $1 \leq k \leq n$, we write $\alpha \pm e_{k}$ for $\left(\alpha_{1}, \ldots, \alpha_{k} \pm 1, \ldots, \alpha_{n}\right)$.

Let $n \geq 0$ and let $\beta \in H_{2}^{+}(X)$ be a non-zero homology class. We denote by $\bar{M}_{n}(X, \beta):=\bar{M}_{0, n}(X, \beta)$ the Deligne-Mumford stack of $n$-pointed genus zero stable maps to $X$ of class $\beta$ as defined in BM].

The moduli space $\bar{M}_{\alpha}^{Y}(X, \beta)$ that we want to construct should be thought of as a compactification of the space of all irreducible stable maps $\left(\mathbb{P}^{1}, x_{1}, \ldots, x_{n}, f\right)$ to $X$ of class $\beta$ that meet $Y$ in the points $x_{i}$ with multiplicity $\alpha_{i}$ for all $i$. We define it first as a subset of the set of geometric points of $\bar{M}_{n}(X, \beta)$, but we will see soon that it has the structure of a closed substack of $\bar{M}_{n}(X, \beta)$.

Definition 1.1. With notations as above, we define $\bar{M}_{\alpha}^{Y}(X, \beta)$ to be the locus in $\bar{M}_{n}(X, \beta)$ of all stable maps $\left(C, x_{1}, \ldots, x_{n}, f\right)$ such that 
(i) $f\left(x_{i}\right) \in Y$ for all $i$ with $\alpha_{i}>0$,

(ii) $f^{*} Y-\sum_{i} \alpha_{i} x_{i} \in A_{0}\left(f^{-1}(Y)\right)$ is effective.

If there is no risk of confusion we will write $\bar{M}_{\alpha}(X, \beta)$ instead of $\bar{M}_{\alpha}^{Y}(X, \beta)$.

Remark 1.2. Condition (i) is obviously necessary for [ii] to make sense. The cycle class $f^{*} Y \in A_{0}\left(f^{-1}(Y)\right)$ is well-defined by [F] chapter 6 as the intersection product $Y \cdot C$ in $Y \times{ }_{X} C=f^{-1}(Y)$. Note that the Chow groups of a scheme are equal to the Chow groups of its underlying reduced scheme (see [F] example 1.3.1 (a)), so we may replace $f^{-1}(Y)$ by its underlying reduced scheme above. So, by abuse of notation, if we talk about connected (resp. irreducible) components of $f^{-1}(Y)$ in the sequel we will always mean connected (resp. irreducible) components of the underlying reduced scheme of $f^{-1}(Y)$.

Remark 1.3. For degree reasons, the space $\bar{M}(X, \beta)$ is obviously empty if $\sum \alpha>$ $Y \cdot \beta$, so we will tacitly assume from now on that $\sum \alpha \leq Y \cdot \beta$.

Remark 1.4. The Chow group $A_{0}$ of a point as well as of (connected but not necessarily irreducible) genus zero curves is just $\mathbb{Z}$, so condition (ii) in definition 1.1 can be reformulated as follows: for any connected component $Z$ of $f^{-1}(Y)$ we must have

(i) if $Z$ is a point, it is either unmarked or a marked point $x_{i}$ such that the multiplicity of $f$ at $x_{i}$ along $Y$ is at least $\alpha_{i}$,

(ii) if $Z$ is one-dimensional, let $C^{(i)}$ for $1 \leq i \leq r$ be the irreducible components of $C$ not in $Z$ but intersecting $Z$, and let $m^{(i)}$ be the multiplicity of $\left.f\right|_{C^{(i)}}$ at $Z \cap C^{(i)}$ along $Y$. Then we must have

$$
Y \cdot f_{*} Z+\sum_{i=1}^{r} m^{(i)} \geq \sum_{x_{i} \in Z} \alpha_{i} .
$$

Example 1.5. Let $X=\mathbb{P}^{3}, Y=H$ a plane, $\beta=5 \cdot$ [line], and $\alpha=(1,2)$. In the following picture, the curve on the left is in $\bar{M}_{(1,2)}(X, \beta)$, whereas the one on the right is not (condition (ii) of remark 1.4 is violated for the line marked $Z$, as $1+1 \nsupseteq 2+1)$.
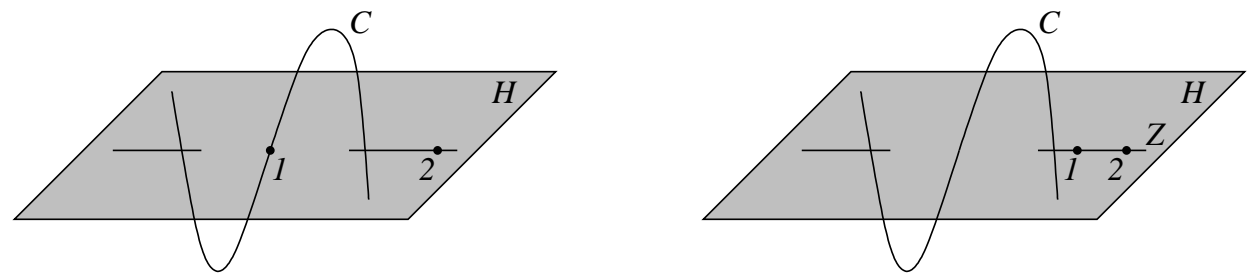

The first thing we will do is to study the space $\bar{M}_{\alpha}(X, \beta)$ in the special case where $X=\mathbb{P}^{N}$ and $Y=H$ is a hyperplane. In this case, we will write $\bar{M}_{\alpha}(X, \beta)$ as $\bar{M}_{\alpha}\left(\mathbb{P}^{N}, d\right)$, where $d=H \cdot \beta$. The main result of this section is that the general element of $\bar{M}_{\alpha}\left(\mathbb{P}^{N}, d\right)$ corresponds to an irreducible stable map whose image is not contained in $H$, i.e. that the curves in $\bar{M}_{\alpha}\left(\mathbb{P}^{N}, d\right)$ are exactly those that can be deformed to an irreducible curve that still satisfies the given multiplicity conditions and that is not contained in $H$. (Here and in the following, by "the curve $\mathcal{C}$ can be deformed to a curve satisfying a property $P$ " we mean that there is a family of stable maps such that the central fiber is $\mathcal{C}$ and the general fiber has $P$.) 
Definition 1.6. We define $M_{\alpha}\left(\mathbb{P}^{N}, d\right)$ to be the subset of $\bar{M}_{\alpha}\left(\mathbb{P}^{N}, d\right)$ of all stable maps $\left(C, x_{1}, \ldots, x_{n}, f\right)$ with $C \cong \mathbb{P}^{1}$ and $f(C) \not \subset H$.

Remark 1.7. We will often consider first the easier case of the spaces $\bar{M}_{\alpha}\left(\mathbb{P}^{N}, d\right)$ with the additional condition that $\sum \alpha=d$. (This is the situation that has been studied in [V].) In this case, condition [ii] in definition 1.1 actually means that $f^{*} H-\sum_{i} \alpha_{i} x_{i}=0 \in A_{0}\left(f^{-1}(H)\right)$. Correspondingly, the conditions in remark 1.4 read as follows: for any connected component $Z$ of $f^{-1}(H)$ we must have

(i) if $Z$ is a point, it is a marked point $x_{i}$ with $\alpha_{i}$ being equal to the multiplicity of $f$ at $x_{i}$ along $H$,

(ii) if $Z$ is one-dimensional, let $C^{(i)}$ for $1 \leq i \leq r$ be the irreducible components of $C$ not in $Z$ but intersecting $Z$, and let $m^{(i)}$ be the multiplicity of $\left.f\right|_{C^{(i)}}$ at $Z \cap C^{(i)}$ along $H$. Then we must have

$$
\left.\operatorname{deg} f\right|_{Z}+\sum_{i=1}^{r} m^{(i)}=\sum_{x_{i} \in Z} \alpha_{i} .
$$

Lemma 1.8. The space $M_{\alpha}\left(\mathbb{P}^{N}, d\right)$ has the structure of an irreducible and locally closed substack of $\bar{M}_{n}\left(\mathbb{P}^{N}, d\right)$.

Proof. The locus of irreducible stable maps $\left(\mathbb{P}^{1}, x_{1}, \ldots, x_{n}, f\right) \in \bar{M}_{n}\left(\mathbb{P}^{N}, d\right)$ such that $f\left(\mathbb{P}^{1}\right) \not \subset H$ can be written as $M_{n}\left(\mathbb{P}^{N}, d\right) \backslash \bar{M}_{n}(H, d)$, so it is open in $\bar{M}_{n}\left(\mathbb{P}^{N}, d\right)$. On the other hand, the condition that $f$ vanishes to order at least $\alpha_{i}$ along $H$ at $x_{i}$ is closed, so $M_{\alpha}\left(\mathbb{P}^{N}, d\right)$ is the intersection of a closed subset with an open subset in $\bar{M}_{n}\left(\mathbb{P}^{N}, d\right)$. It is irreducible as there is a surjective rational map

$$
\begin{aligned}
& \mathbb{C}^{2 n} \times H^{0}\left(\mathbb{P}^{1}, \mathcal{O}\left(d-\sum \alpha\right)\right) \times H^{0}\left(\mathbb{P}^{1}, \mathcal{O}(d)\right)^{N} \quad \rightarrow \quad M_{\alpha}\left(\mathbb{P}^{n}, d\right) \\
& \left(a_{1}, b_{1}, \ldots, a_{n}, b_{n}, f_{0}, f_{1}, \ldots, f_{N}\right) \mapsto\left(\mathbb{P}^{1},\left(a_{1}: b_{1}\right), \ldots,\left(a_{n}: b_{n}\right), f\right)
\end{aligned}
$$

where

$$
f(z)=f\left(z_{0}: z_{1}\right)=\left(f_{0}(z) \cdot \prod_{i=1}^{n}\left(z_{1} a_{i}-z_{0} b_{i}\right)^{\alpha_{i}}: f_{1}(z): \cdots: f_{N}(z)\right)
$$

whose domain space is irreducible.

Lemma 1.9. The closure of $M_{\alpha}\left(\mathbb{P}^{N}, d\right)$ in $\bar{M}_{n}\left(\mathbb{P}^{N}, d\right)$ is contained in $\bar{M}_{\alpha}\left(\mathbb{P}^{N}, d\right)$.

Proof. This follows from the continuity of intersection products. To be more precise, let $\mathcal{C}$ be a point in the closure of $M_{\alpha}\left(\mathbb{P}^{N}, d\right)$. By lemma 1.8 there is a family $\phi: T \rightarrow \bar{M}_{n}\left(\mathbb{P}^{N}, d\right)$ of stable maps over a smooth curve $T$ with a distinguished point $0 \in T$ such that $\phi(0)=\mathcal{C}$ and $\phi(t) \in M_{\alpha}\left(\mathbb{P}^{N}, d\right)$ for $t \neq 0$. We have to prove that $\phi(0) \in \bar{M}_{\alpha}\left(\mathbb{P}^{N}, d\right)$. As it is obvious that $\phi(0)$ satisfies condition (i) of definition 1.1, it remains to show (ii).

The family $\phi$ is given by the data $\left(C, x_{1}, \ldots, x_{n}, f\right)$ where $\pi: C \rightarrow T$ is a curve over $T$, the $x_{i}: T \rightarrow C$ are sections of $\pi$, and $f: C \rightarrow \mathbb{P}^{N}$ is a morphism. Set $C_{H}=f^{-1}(H)$ and consider the 1-cycles $f^{*} H$ and $\sum_{i} \alpha_{i} x_{i}(T)$ in $A_{1}\left(C_{H}\right)$. By assumption, the cycle $\gamma:=f^{*} H-\sum_{i} \alpha_{i} x_{i}(T)$ is effective (it might however have components over $0 \in T$ coming from $f^{*} H$ ). Applying [F] proposition 11.1 (b) to the cycles $f^{*} H$ and $\gamma+\sum_{i} \alpha_{i} x_{i}(T)$ we see that the specialization of $f^{*} H$ at $t=0$ is equal to the limit cycle of $\gamma+\sum_{i} \alpha_{i} x_{i}(T)$ as $t \rightarrow 0$. As the limit cycle of $\gamma$ for $t \rightarrow 0$ is effective, we have shown that $\phi(0)$ satisfies [ii). This shows the lemma. 
Definition 1.10. Let $\mathcal{C}=\left(C, x_{1}, \ldots, x_{n}, f\right) \in \bar{M}_{\alpha}\left(\mathbb{P}^{N}, d\right)$ be a stable map. An irreducible component $Z$ of $C$ is called an internal component of $\mathcal{C}$ if $f(C) \subset$ $H$, and an external component otherwise. A subcurve of $\mathcal{C}$ is a stable map $\mathcal{C}^{\prime}=$ $\left(C^{\prime}, x_{1}^{\prime}, \ldots, x_{m}^{\prime}, f^{\prime}\right) \in \bar{M}_{\alpha^{\prime}}\left(\mathbb{P}^{N}, d^{\prime}\right)$ constructed from $\mathcal{C}$ as follows. Let $C^{\prime}$ be any proper connected subcurve of $C$, and let $f^{\prime}=\left.f\right|_{C^{\prime}}$. The marked points $x_{1}^{\prime}, \ldots, x_{m}^{\prime}$ are the marked points $x_{i}$ contained in $C^{\prime}$, together with all the intersection points of $C^{\prime}$ with the other irreducible components of $C$. We assign multiplicities $\alpha^{\prime}=$ $\left(\alpha_{1}^{\prime}, \ldots, \alpha_{m}^{\prime}\right)$ to the points $x_{1}^{\prime}, \ldots, x_{m}^{\prime}$ as follows: The points $x_{i}$ on $C^{\prime}$ will have their given multiplicity $\alpha_{i}$. The intersection points with other irreducible components of $C$ will be assigned the multiplicity of $f^{\prime}$ along $H$ at that point if the point lies on an external component of $C^{\prime}$, and 0 otherwise. Let $d^{\prime}$ be the degree of $f^{\prime}$ on $C^{\prime}$. The following picture shows an example of this construction, where the marked points are labeled with their multiplicities.
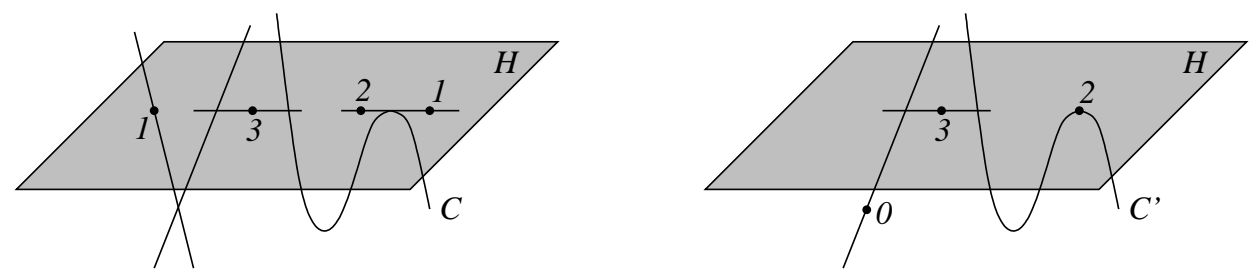

Lemma 1.11. Let $\mathcal{C} \in \bar{M}_{\alpha}\left(\mathbb{P}^{N}, d\right)$ be a stable map and assume that $\sum \alpha=d$. Let $\mathcal{C}^{\prime}=\left(C^{\prime}, x_{1}^{\prime}, \ldots, x_{n}^{\prime}, f^{\prime}\right)$ be a subcurve of $\mathcal{C}$ with the following property: if $Z$ is an internal irreducible component of $C$ contained in $C^{\prime}$, then any adjacent irreducible component of $Z$ in $C$ is also contained in $C^{\prime}$. (For example, the subcurve in the picture above satisfies this property.) Then $\sum \alpha^{\prime}=d^{\prime}$.

Proof. The condition $\sum \alpha=d$ means that $f^{*} H-\sum \alpha_{i} x_{i}=0 \in A_{0}\left(f^{-1}(H)\right)$. We claim that also $f^{\prime *} H-\sum \alpha_{i}^{\prime} x_{i}^{\prime}=0 \in A_{0}\left(f^{\prime-1}(H)\right)$, which then implies that $\sum \alpha^{\prime}=d^{\prime}$. In fact, this can be checked on the connected components of $f^{\prime-1}(H)$. Let $Z$ be a connected component of $f^{\prime-1}(H)$. By assumption, there are only two possibilities:

- $C$ and $C^{\prime}$ are locally isomorphic in a neighborhood of $Z$, i.e. $Z$ is also a connected component of $f^{-1}(H)$. Therefore, $\left.\left(f^{\prime *} H-\sum \alpha_{i}^{\prime} x_{i}^{\prime}\right)\right|_{Z}=0 \in A_{0}(Z)$.

- $Z$ is an intersection point of $C^{\prime}$ with $\overline{C \backslash C^{\prime}}$ that lies on an external component of $C^{\prime}$. Then, by definition of a subcurve, $Z$ is a marked point of $\mathcal{C}^{\prime}$ with multiplicity equal to the multiplicity of $f^{\prime}$ along $H$ at $Z$. In particular, we have again that $\left.\left(f^{\prime *} H-\sum \alpha_{i}^{\prime} x_{i}^{\prime}\right)\right|_{Z}=0 \in A_{0}(Z)$.

This proves the lemma.

Lemma 1.12. A stable map $\mathcal{C}=\left(C, x_{1}, \ldots, x_{n}, f\right) \in \bar{M}_{\alpha}\left(\mathbb{P}^{N}, d\right)$ can be deformed to an irreducible curve in $\bar{M}_{\alpha}\left(\mathbb{P}^{N}, d\right)$ if one of the following conditions is satisfied:

(i) $C$ has only internal components.

(ii) $\sum \alpha=d$, and $C$ consists exactly of one internal component $C^{(0)}$ and $r$ external components $C^{(1)}, \ldots, C^{(r)}$ intersecting $C^{(0)}$ for some $r \geq 0$ (i.e. $C$ is a "comb", with the central component being internal and the teeth external, see the picture in construction 2.1). Moreover, in this case $\mathcal{C}$ can even be deformed to an irreducible curve that is not contained in $H$ (which is then obvious unless $r=0$ ). 
(iii) $\sum \alpha=d$, and $C$ has exactly two irreducible components $C^{(1)}$ and $C^{(2)}$, both being external.

Proof. To show (i), note that by definition every curve with $f(C) \subset H$ lies in $\bar{M}_{\alpha}\left(\mathbb{P}^{N}, d\right)$, so $\bar{M}_{n}(H, d) \subset \bar{M}_{\alpha}\left(\mathbb{P}^{N}, d\right)$. But it is well-known that the space of irreducible curves inside $\bar{M}_{n}(H, d)$ is dense, so $\mathcal{C}$ can be deformed to an irreducible curve in $\bar{M}_{\alpha}\left(\mathbb{P}^{N}, d\right)$.

(11) has been shown in $[\mathrm{V}$ theorem 6.1. (In fact, in the notations used in $[\mathrm{V}]$, our curve $\mathcal{C}$ is an element of a space $\mathcal{Y}$ with suitable decorations as introduced in [V] definition 3.7.)

Finally, in the case (iii) it is easy to construct an explicit deformation. Choose homogeneous coordinates $z_{0}, \ldots, z_{N}$ on $\mathbb{P}^{N}$ such that $H$ is given by the equation $z_{0}=0$. The map $f: C \rightarrow \mathbb{P}^{N}$ is then given by sections $s_{0}, \ldots, s_{N}$ of a suitable line bundle $\mathcal{L}$ on $C$. We may assume that the coordinates are chosen such that the $s_{i}$ do not vanish at $C^{(1)} \cap C^{(2)}$ (as for $s_{0}$ note that $s_{0}\left(C^{(1)} \cap C^{(2)}\right.$ ) $=0$ would mean that the intersection point lies on $H$, so it must be a marked point by remark 1.7 (i), hence it must be non-singular, which is a contradiction). Let $D_{i}=\left(s_{i}\right)$ be the associated divisors, in particular $D_{0}=\sum \alpha_{i} x_{i}$.

Now let $W$ be the blow-up of $\mathbb{C} \times \mathbb{P}^{1}$ at the point $(0,0)$, considered as a onedimensional family of curves by the projection map $\pi: W \rightarrow \mathbb{C}$. We can identify the fiber $\pi^{-1}(0)$ with $C^{(1)} \cup C^{(2)}$. The points $x_{i} \in \pi^{-1}(0)$ can be extended to sections $\tilde{x}_{i}$ of $\pi$, giving rise to an extended divisor $\tilde{D}_{0}=\sum \alpha_{i} \tilde{x}_{i}$. In the same way one can find divisors $\tilde{D}_{i}$ on $W$ such that $\left.\tilde{D}_{i}\right|_{\pi^{-1}(0)}=D_{i}$ for all $i$. As Pic $W=\operatorname{Pic} C$, these divisors will be linearly equivalent and define a line bundle $\tilde{\mathcal{L}}$ on $W$ such that $\left.\tilde{\mathcal{L}}\right|_{\pi^{-1}(0)}=\mathcal{L}$. Moreover, after possibly restricting the base $\mathbb{C}$ to a smaller open neighborhood of 0 we can assume that the $\tilde{D}_{i}$ are base-point free. Finally, we can choose sections $\tilde{s}_{i}$ of $\tilde{\mathcal{L}}$ such that $\left(\tilde{s}_{i}\right)=\tilde{D}_{i}$ and $\tilde{s}_{i} \mid \pi^{-1}(0)=s_{i}$. Then $\left(W, \tilde{x}_{0}, \ldots, \tilde{x}_{n},\left(\tilde{s}_{0}: \cdots: \tilde{s}_{N}\right)\right)$ is a family of stable maps whose central fiber is $\mathcal{C}$ and whose general element is in $M_{\alpha}\left(\mathbb{P}^{N}, d\right)$.

Lemma 1.13. Let $\mathcal{C}=\left(C, x_{1}, \ldots, x_{n}, f\right) \in \bar{M}_{\alpha}\left(\mathbb{P}^{N}, d\right)$ be a reducible stable map and assume that $\sum \alpha=d$. Then $\mathcal{C}$ can be deformed to a stable map in $\bar{M}_{\alpha}\left(\mathbb{P}^{N}, d\right)$ with fewer nodes.

Proof. This is essentially obtained from lemma 1.12 by gluing. Pick a node $P \in C$ and a subcurve $\mathcal{C}^{(0)}=\left(C^{(0)}, x_{1}^{(0)}, \ldots, x_{n^{(0)}}^{(0)}, f^{(0)}\right) \in \bar{M}_{\alpha^{(0)}}\left(\mathbb{P}^{N}, d^{(0)}\right)$ of $\mathcal{C}$ as follows:

(i) If $C$ has a node connecting two internal components of $C$, let $P$ be this node and let $C^{(0)}$ be the connected component of $f^{-1}(H)$ containing $P$.

(ii) Otherwise, if $C$ has a node connecting an internal component $Z$ to an external component of $C$, let $P$ be this node and let $C^{(0)}$ be $Z$ together with all adjacent (necessarily external) components of $C$.

(iii) Otherwise, let $P$ be any node of $C$ (necessarily connecting two external components of $C$ ) and let $C^{(0)}$ be the two irreducible components of $C$ meeting at $P$.

Let $C^{(1)}, \ldots, C^{(r)}$ with $r \geq 0$ be the connected components of $\overline{C \backslash C^{(0)}}$.

In any case, we can deform $\mathcal{C}^{(0)}$ to an irreducible map in $\bar{M}_{\alpha^{(0)}}\left(\mathbb{P}^{N}, d^{(0)}\right)$ by lemma 1.12 (in the cases (ii) and (iii) it follows from lemma 1.11 that $\sum \alpha^{(0)}=d^{(0)}$ ). So let $\phi: T \rightarrow \bar{M}_{\alpha^{(0)}}\left(\mathbb{P}^{N}, d^{(0)}\right)$ be a deformation of $\mathcal{C}^{(0)}$ for some smooth pointed curve $(T, 0)$, i.e. $\phi(0)=\mathcal{C}^{(0)}$ and for all $0 \neq t \in T$ the curve $\phi(t)$ is irreducible. 
This deformation is given by a family $\pi: \tilde{C} \rightarrow T$ of curves, sections $\tilde{x}_{1}, \ldots, \tilde{x}_{n}$ of $\pi$ and a map $\tilde{f}: \tilde{C} \rightarrow \mathbb{P}^{N}$. For all $1 \leq i \leq r$, the intersection point of $C^{(0)}$ and $C^{(i)}$ is one of the marked points of $C^{(0)}$, hence corresponds to a marked point of $\phi$, say $\tilde{x}_{i}$. Note that in all cases (i) to (iii) above, the deformation $\phi$ has the property that $\tilde{f}\left(\tilde{x}_{i}(t)\right) \in H$ for all $t \in T$ if this is true for $t=0$. In particular, there are $T$-valued projective automorphisms $\psi_{i}: T \rightarrow$ PGL $(N)$ keeping $H$ fixed such that $\psi_{i}(t)\left(\tilde{f}\left(\tilde{x}_{i}(0)\right)\right)=\tilde{f}\left(\tilde{x}_{i}(t)\right)$. The induced action of PGL $(N)$ on the moduli spaces $\bar{M}_{\alpha^{(i)}}\left(\mathbb{P}^{N}, d^{(i)}\right)$ makes $\psi_{i}$ into a deformation of $\mathcal{C}^{(i)}$ over $T$ such that for all $t \in T$ the marked point corresponding to $C^{(0)} \cap C^{(i)}$ is mapped to the same point in $\mathbb{P}^{N}$ by the families $\phi$ and $\psi_{i}$. This means that the families $\phi$ and $\psi_{i}$ can actually be glued to give a deformation of the original curve $\mathcal{C}$. This deformation smoothes the node $P$.

Proposition 1.14. The closure of $M_{\alpha}\left(\mathbb{P}^{N}, d\right)$ in $\bar{M}_{n}\left(\mathbb{P}^{N}, d\right)$ is equal to $\bar{M}_{\alpha}\left(\mathbb{P}^{N}, d\right)$. In particular, $\bar{M}_{\alpha}\left(\mathbb{P}^{N}, d\right)$ has the structure of an irreducible, proper, reduced substack of $\bar{M}_{n}\left(\mathbb{P}^{N}, d\right)$.

Proof. " $\subset$ " has been shown in lemma 1.9, so it remains to show " $\supset$ ". Let $\mathcal{C} \in$ $\bar{M}_{\alpha}\left(\mathbb{P}^{N}, d\right)$ be a stable map. Assume first that $\sum \alpha=d$. Using lemma 1.13 inductively, we can deform $\mathcal{C}$ to an irreducible curve in $\bar{M}_{\alpha}\left(\mathbb{P}^{N}, d\right)$. If this irreducible curve does not lie inside $H$ then we are done, otherwise use the $r=0$ case of lemma 1.12 (ii).

If $k=d-\sum \alpha>0$, let $\alpha^{\prime}=\alpha \cup(1, \ldots, 1)$ such that $\sum \alpha^{\prime}=d$. By adding marked points (and possibly introducing new contracted components) it is easy to find a stable map $\mathcal{C}^{\prime} \in \bar{M}_{\alpha^{\prime}}$ that maps to $\mathcal{C}$ under the forgetful morphism $\bar{M}_{n+k}\left(\mathbb{P}^{N}, d\right) \rightarrow$ $\bar{M}_{n}\left(\mathbb{P}^{N}, d\right)$. By the above, $\mathcal{C}^{\prime}$ can be deformed to an irreducible curve in $M_{\alpha^{\prime}}\left(\mathbb{P}^{N}, d\right)$, which induces a deformation of $\mathcal{C}$ to an irreducible curve in $M_{\alpha}\left(\mathbb{P}^{N}, d\right)$.

Hence we finally have shown that $\bar{M}_{\alpha}\left(\mathbb{P}^{N}, d\right)$ is closed. So by giving it the reduced substack structure, we get a proper, reduced substack of $\bar{M}_{n}\left(\mathbb{P}^{N}, d\right)$ which is irreducible by lemma 1.8 .

Lemma 1.15. The moduli space $\bar{M}_{\alpha}\left(\mathbb{P}^{N}, d\right)$ has the following properties:

(i) If $k=d-\sum \alpha>0$ and we let $\alpha^{\prime}=\alpha \cup(1, \ldots, 1)$ such that $\sum \alpha^{\prime}=d$, then there is a degree- $k$ ! generically finite cover $\bar{M}_{\alpha^{\prime}}\left(\mathbb{P}^{N}, d\right) \rightarrow \bar{M}_{\alpha}\left(\mathbb{P}^{N}, d\right)$, given by forgetting the last $k$ marked points and stabilizing.

(ii) $\bar{M}_{\alpha \cup(0)}\left(\mathbb{P}^{N}, d\right)$ is the universal curve over $\bar{M}_{\alpha}\left(\mathbb{P}^{N}, d\right)$. In particular, if $\alpha=$ $(0, \ldots, 0)$ then $\bar{M}_{\alpha}\left(\mathbb{P}^{N}, d\right)=\bar{M}_{|\alpha|}\left(\mathbb{P}^{N}, d\right)$.

(iii) The moduli space $\bar{M}_{\alpha}\left(\mathbb{P}^{N}, d\right)$ is purely of the expected dimension, which is $\operatorname{dim} \bar{M}_{|\alpha|}\left(\mathbb{P}^{N}, d\right)-\sum \alpha=d(N+1)+N-3+|\alpha|-\sum \alpha$.

Proof. To show (i), note that from the parametrization of $M_{\alpha}\left(\mathbb{P}^{N}, d\right)$ given in the proof of lemma 1.8 one can see that the general element of $M_{\alpha}\left(\mathbb{P}^{N}, d\right)$ corresponds to a stable map $\left(\mathbb{P}^{1}, x_{1}, \ldots, x_{n}, f\right)$ such that $f^{*} H$ is equal to $\sum_{i} \alpha_{i} x_{i}$ plus a union of $k=d-\sum \alpha_{i}$ distinct unmarked points with multiplicity one. Obviously, the map $\bar{M}_{\alpha^{\prime}}\left(\mathbb{P}^{N}, d\right) \rightarrow \bar{M}_{\alpha}\left(\mathbb{P}^{n}, d\right)$ is finite over these elements, and it has degree $k$ !, corresponding to the choice of order of the $k$ added marked points.

As in the proof of (i), the statement of (ii) is obvious on the dense open subset of $\bar{M}_{\alpha}\left(\mathbb{P}^{N}, d\right)$ described above, and it extends to the closures because of the flatness of the map $\bar{M}_{n+1}\left(\mathbb{P}^{N}, d\right) \rightarrow \bar{M}_{n}\left(\mathbb{P}^{N}, d\right)$. 
Finally, (iii) has been shown in $\mathrm{V}$ | proposition 5.7 if $\sum \alpha=d$. Otherwise use (i) first. Alternatively, (iii) can be read off from the parametrization given in the proof of lemma 1.8 .

Remark 1.16. The stack $\bar{M}_{\alpha}\left(\mathbb{P}^{N}, d\right)$ is in general singular, even in codimension one (see $\llbracket \mathrm{V}$ corollary 4.16). However, it is smooth at all points $\left(\mathbb{P}^{1}, x_{1}, \ldots, x_{n}, f\right) \in$ $M_{\alpha}\left(\mathbb{P}^{N}, d\right)$. In fact, for these curves the obstruction space for deformations inside $\bar{M}_{\alpha}\left(\mathbb{P}^{N}, d\right)$ is $H^{1}\left(\mathbb{P}^{1}, f^{*} T_{\mathbb{P}^{N}}^{\prime}\right)$, where $f^{*} T_{\mathbb{P}^{N}}^{\prime}$ is the kernel of the composite map

$$
\left.f^{*} T_{\mathbb{P}^{N}} \rightarrow f^{*} N_{H / \mathbb{P}^{N}} \rightarrow\left(f^{*} N_{H / \mathbb{P}^{N}}\right)\right|_{Z}
$$

with $Z$ being the zero-dimensional subscheme of $\mathbb{P}^{1}$ having length $\alpha_{i}$ at the point $x_{i}$ for all $i$. But as both these maps are surjective on global sections (for the second one note that $f^{*} N_{H / \mathbb{P}^{N}}=\mathcal{O}(d)$ and $\left.\sum \alpha \leq d\right)$, it follows that $H^{1}\left(\mathbb{P}^{1}, f^{*} T_{\mathbb{P}^{N}}^{\prime}\right)=0$.

However, we will not need any smoothness results in our paper.

Now we return to the general case of the moduli space $\bar{M}_{n}^{Y}(X, \beta)$ where $X$ is any smooth projective variety and $Y \subset X$ a smooth very ample hypersurface. One of the main problems is that these spaces will in general not have the expected dimension. This means in particular that we need virtual fundamental classes, which cannot be obtained using the techniques above. To overcome this problem, we use the linear system $|Y|$ to get a map $X \rightarrow \mathbb{P}^{N}$, and consider the space $\bar{M}_{\alpha}^{Y}(X, \beta)$ as the "intersection" of two problems we already know: (a) stable maps in $X$ and (b) stable maps in $\mathbb{P}^{N}$ with given multiplicities to the hyperplane $H \subset \mathbb{P}^{N}$ induced by $Y$.

We fix the following notation: let $\varphi: X \rightarrow \mathbb{P}^{N}$ be the morphism determined by $|Y|$, and let $H \subset \mathbb{P}^{N}$ the hyperplane such that $Y=\varphi^{-1}(H)$. As $d:=Y \cdot \beta>0$, the map $\varphi$ induces a morphism $\phi: \bar{M}_{n}(X, \beta) \rightarrow \bar{M}_{n}\left(\mathbb{P}^{N}, d\right)$ (see BM]).

Remark 1.17. Let $\mathcal{C} \in \bar{M}_{n}(X, \beta)$. As the conditions (i) and (ii) of definition 1.1 pull back nicely, it is obvious that $\mathcal{C} \in \bar{M}_{\alpha}^{Y}(X, \beta)$ if and only if $\phi(\mathcal{C}) \in \bar{M}_{\alpha}^{H}\left(\mathbb{P}^{N}, d\right)$.

Definition 1.18. By the previous remark, the space $\bar{M}_{\alpha}^{Y}(X, \beta)$ has the structure of a proper closed substack of $\bar{M}_{n}(X, \beta)$ by requiring the diagram of inclusions

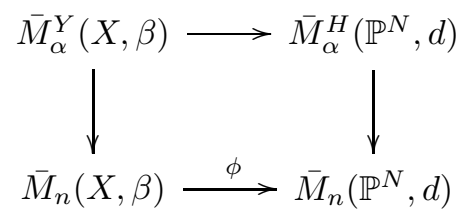

to be cartesian. We define the virtual fundamental class $\left[\bar{M}_{\alpha}^{Y}(X, \beta)\right]^{\text {virt }}$ to be the one induced by the virtual fundamental class of $\bar{M}_{n}(X, \beta)$ (see e.g. $[\mathrm{B}, \sqrt[\mathrm{BF}]{ }$ ) and the usual fundamental class of $\bar{M}_{\alpha}^{H}\left(\mathbb{P}^{N}, d\right)$, in the sense of the following remark.

Remark 1.19. Let $M_{1}$ and $M_{2}$ be Deligne-Mumford stacks over a smooth DeligneMumford stack $S$. Let $M=M_{1} \times_{S} M_{2}$, so that we have a cartesian diagram

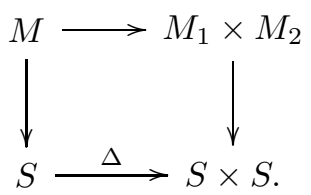


Assume that we are given classes $\gamma_{1} \in A_{*}\left(M_{1}\right)$ and $\gamma_{2} \in A_{*}\left(M_{2}\right)$ (usually thought of as virtual fundamental classes in this paper). Then the class $\Delta^{!}\left(\gamma_{1} \otimes \gamma_{2}\right)$ in $M$ will be called induced by $\gamma_{1}$ and $\gamma_{2}$. If the maps $M_{1} \rightarrow S$ and $M_{2} \rightarrow S$ are inclusions, this is actually the usual refined intersection product of $\gamma_{1}$ and $\gamma_{2}$. This is the case in the above definition, but we mentioned the general case here as we will need it later on.

By lemma 1.15 (iii), the virtual fundamental class of $\bar{M}_{\alpha}^{Y}(X, \beta)$ defined above has dimension $\operatorname{dim} \bar{M}_{n}(X, \beta)-\sum \alpha$, which is the expected dimension of $\bar{M}_{\alpha}^{Y}(X, \beta)$. If $X$ is a projective space and $Y \subset X$ a hyperplane, it is obvious by definition that the virtual fundamental class of $\bar{M}_{\alpha}^{Y}(X, \beta)$ is equal to the usual one.

\section{INCREASING THE MULTIPLICITIES}

By construction, $\bar{M}_{\alpha+e_{k}}(X, \beta)$ is a closed substack of $\bar{M}_{\alpha}(X, \beta)$ of expected codimension one. The main goal of this paper is to compute $\left[\bar{M}_{\alpha+e_{k}}(X, \beta)\right]^{\text {virt }}$ as a cycle in the Chow group of $\bar{M}_{\alpha}(X, \beta)$. We start with the following naïve approach describing the transition from multiplicity $\alpha_{k}$ to $\alpha_{k}+1$ at the point $x_{k}$.

Construction 2.1. Consider a moduli space $M=\bar{M}_{n}(X, \beta)$ and let $\mathcal{C} \rightarrow M$ be the universal curve, with evaluation map ev $: \mathcal{C} \rightarrow X$. Fix $k$ with $1 \leq k \leq n$ and let $s_{k}: M \rightarrow \mathcal{C}$ denote the section corresponding to the marked point $x_{k}$. Let $y \in H^{0}\left(\mathcal{O}_{X}(Y)\right)$ be the equation of $Y$. Choose an integer $m \geq 0$. We pull $y$ back to $\mathcal{C}$ by $e v$, take the $m$-jet relative to $M$ of it and pull this back to $M$ by $s_{k}$ to get a section

$$
\sigma_{k}^{m}:=s_{k}^{*} d_{\mathcal{C} / M}^{m} e v^{*} y \in H^{0}\left(M, s_{k}^{*} \mathcal{P}_{\mathcal{C} / M}^{m}\left(e v^{*} \mathcal{O}_{X}(Y)\right)\right),
$$

where $\mathcal{P}_{\mathcal{C} / M}^{m}\left(e v^{*} \mathcal{O}_{X}(Y)\right)$ denotes relative principal parts of order $m$ (or $m$-jets) of the line bundle $e v^{*} \mathcal{O}_{X}(Y)$, and $d_{\mathcal{C} / M}^{m}$ is the derivative up to order $m$ (see [EGA4] 16.3, 16.7.2.1 for precise definitions). Geometrically, $\sigma_{k}^{m}$ vanishes precisely on the stable maps that have multiplicity at least $m+1$ to $Y$ at the point $x_{k}$. By [EGA4] 16.10.1, 16.7.3 there is an exact sequence

$$
0 \rightarrow L_{k}^{\otimes m} \otimes e v_{k}^{*} \mathcal{O}_{X}(Y) \rightarrow s_{k}^{*} \mathcal{P}_{\mathcal{C} / M}^{m}\left(e v^{*} \mathcal{O}_{X}(Y)\right) \rightarrow s_{k}^{*} \mathcal{P}_{\mathcal{C} / M}^{m-1}\left(e v^{*} \mathcal{O}_{X}(Y)\right) \rightarrow 0
$$

where we set $\mathcal{P}_{\mathcal{C} / M}^{-1}\left(e v^{*} \mathcal{O}_{X}(Y)\right)=0$, and where $L_{k}=s_{k}^{*} \omega_{\mathcal{C} / M}$ is the $k$-th cotangent line, i.e. the line bundle on $M$ whose fiber at a point $\left(C, x_{1}, \ldots, x_{n}, f\right)$ is $T_{C, x_{k}}^{\vee}$. Note that the last map in this sequence sends $\sigma_{k}^{m}$ to $\sigma_{k}^{m-1}$ for $m>0$. Now restrict these bundles and sections to $\bar{M}_{\alpha}(X, \beta)$. As all stable maps in $\bar{M}_{\alpha}(X, \beta)$ have multiplicity (at least) $\alpha_{k}$ at $x_{k}$, the restriction of $\sigma_{k}^{\alpha_{k}}$ to $\bar{M}_{\alpha}(X, \beta)$ defines a section

$$
\sigma_{k}:=\left.\sigma_{k}^{\alpha_{k}}\right|_{\bar{M}_{\alpha}(X, \beta)} \in H^{0}\left(L_{k}^{\otimes \alpha_{k}} \otimes e v_{k}^{*} \mathcal{O}_{X}(Y)\right)=H^{0}\left(\mathcal{O}\left(\alpha_{k} \psi_{k}+e v_{k}^{*} Y\right)\right)
$$

on $\bar{M}_{\alpha}(X, \beta)$, where $\psi_{k}=c_{1}\left(L_{k}\right)$.

The vanishing of this section describes exactly the condition that a stable map in $\bar{M}_{\alpha}(X, \beta)$ vanishes up to order $\alpha_{k}+1$ at $x_{k}$. Hence naïvely one would expect that $\bar{M}_{\alpha+e_{k}}(X, \beta)$ is described inside $\bar{M}_{\alpha}(X, \beta)$ by the vanishing of this section, and that $\left[\bar{M}_{\alpha+e_{k}}(X, \beta)\right]^{\text {virt }}$ is given by

$$
\left(\alpha_{k} \psi_{k}+e v_{k}^{*} Y\right) \cdot\left[\bar{M}_{\alpha}(X, \beta)\right]^{v i r t} .
$$


This is not true, however, because of the presence of stable maps with the property that the component on which $x_{k}$ lies is mapped entirely into $Y$. Of course, the section $\sigma_{k}$ vanishes on those stable maps, but they are in general not in $\bar{M}_{\alpha+e_{k}}(X, \beta)$. Hence, these stable maps will also contribute to the expression (11). We will now introduce the moduli spaces of the stable maps occurring in these correction terms. Informally speaking, generic stable maps in these correction terms have $r+1$ irreducible components $C^{(0)}, \ldots, C^{(r)}$ for some $r \geq 0$, where $C^{(0)}$ (called the internal component) is mapped into $Y$, and all $C^{(i)}$ for $i>0$ (called the external components) intersect $C^{(0)}$ and have a prescribed multiplicity $m^{(i)}$ to $Y$ at this intersection point (see the picture below, where $m^{(1)}=1$ and $m^{(2)}=2$ ). The point $x_{k}$ has to lie on $C^{(0)}$. The initial multiplicity conditions $\alpha$ as well as the homology class $\beta$ get distributed in all possible ways to the components $C^{(i)}$.

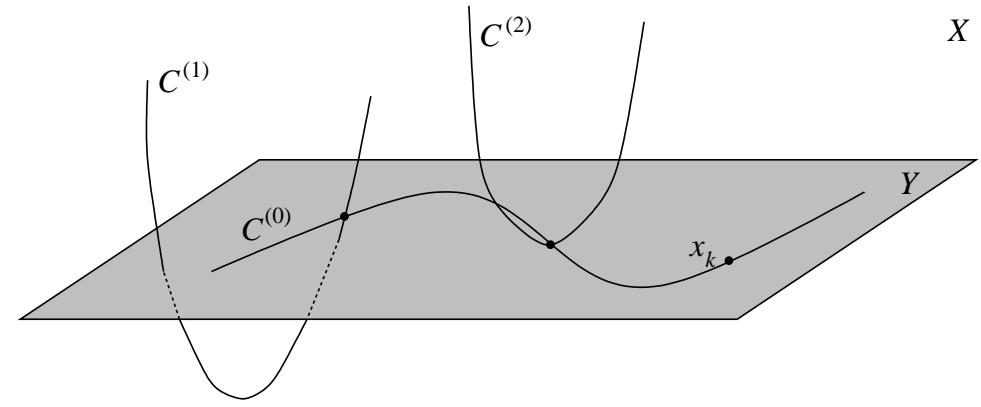

We now describe this more formally.

Definition 2.2. Consider a moduli space $\bar{M}_{\alpha}(X, \beta)$ and $1 \leq k \leq n$ as above. Let $r$ be a non-negative integer. Choose a partition $A=\left(\alpha^{(0)}, \ldots, \alpha^{(r)}\right)$ of $\alpha$ such that $\alpha_{k} \in \alpha^{(0)}$. Let $B=\left(\beta^{(0)}, \ldots, \beta^{(r)}\right)$ be an $(r+1)$-tuple of homology classes with $\beta^{(0)} \in H_{2}^{+}(Y)$ and $\beta^{(i)} \in H_{2}^{+}(X) \backslash\{0\}$ for $i>0$ such that $i_{*} \beta^{(0)}+\beta^{(1)}+$ $\cdots+\beta^{(r)}=\beta$, where $i: Y \rightarrow X$ is the inclusion. Finally, choose an $r$-tuple $M=\left(m^{(1)}, \ldots, m^{(r)}\right)$ of positive integers. With these notations, we define the moduli space $D_{k}(X, A, B, M)$ to be the fiber product

$$
D_{k}(X, A, B, M):=\bar{M}_{\left|\alpha^{(0)}\right|+r}\left(Y, \beta^{(0)}\right) \times_{Y^{r}} \prod_{i=1}^{r} \bar{M}_{\alpha^{(i)} \cup\left(m^{(i)}\right)}\left(X, \beta^{(i)}\right)
$$

where the map from the first factor to $Y^{r}$ is the evaluation at the last $r$ marked points, and the map from the second factor to $Y^{r}$ is the evaluation at the last marked point of each of its factors. We define the virtual fundamental class of $D_{k}(X, A, B, M)$ to be $\frac{m^{(1)} \ldots m^{(r)}}{r !}$ times the class induced by the virtual fundamental classes of its factors, in the sense of remark 1.19. The reason for the unusual multiplicity will become clear in the proof of proposition 3.3 .

Definition 2.3. With notations as in the previous definition, let $D_{\alpha, k}(X, \beta)$ be the disjoint union of the $D_{k}(X, A, B, M)$ for all possible $A, B$, and $M$ satisfying

$$
Y \cdot i_{*} \beta^{(0)}+\sum_{i} m^{(i)}=\sum \alpha^{(0)}
$$

(the reason for this condition will become clear in the following lemma). The virtual fundamental class of $D_{\alpha, k}(X, \beta)$ is defined to be the sum of the virtual fundamental classes of its components $D_{k}(X, A, B, M)$. 
Lemma 2.4. In the case where $X=\mathbb{P}^{N}$ and $Y=H$ is a hyperplane, the moduli spaces $D_{k}\left(\mathbb{P}^{N}, A, B, M\right)$ satisfying equation (国) of definition 2.3 are proper irreducible substacks of $\bar{M}_{\alpha}\left(\mathbb{P}^{N}, d\right)$ of codimension one.

Proof. Considering the definition of the space $D_{k}(X, A, B, M)$, the fact that it is irreducible follows from the following three observations:

(i) $\bar{M}_{\left|\alpha^{(0)}\right|+r}\left(H, d^{(0)}\right)$ is irreducible,

(ii) the evaluation maps $\bar{M}_{\alpha^{(i)} \cup\left(m^{(i)}\right)}\left(\mathbb{P}^{N}, d^{(i)}\right) \rightarrow H$ at the last marked point are flat and surjective (this follows from the action of the group of automorphisms of $\mathbb{P}^{N}$ keeping $H$ fixed on the space $\left.\bar{M}_{\alpha^{(i)} \cup\left(m^{(i)}\right)}\left(\mathbb{P}^{N}, d^{(i)}\right)\right)$,

(iii) the fibers of the maps in (ii) are irreducible (by the Bertini theorem, as the spaces $\bar{M}_{\alpha^{(i)} \cup\left(m^{(i)}\right)}\left(\mathbb{P}^{N}, d^{(i)}\right)$ itself are irreducible by proposition 1.14).

Moreover, these arguments show that the dimension of $D_{k}\left(\mathbb{P}^{N}, A, B, M\right)$ is equal to

$$
\operatorname{dim} \bar{M}_{\left|\alpha^{(0)}\right|+r}\left(H, d^{(0)}\right)+\sum_{i=1}^{r} \operatorname{dim} \bar{M}_{\alpha^{(i)} \cup\left(m^{(i)}\right)}\left(\mathbb{P}^{N}, d^{(i)}\right)-r \cdot(N-1) .
$$

By a quick computation using lemma 1.15)(iii) this is equal to

$$
\operatorname{dim} \bar{M}_{\alpha}\left(\mathbb{P}^{N}, d\right)+\sum \alpha^{(0)}-d^{(0)}-\sum_{i} m^{(i)}-1,
$$

so the dimension statement follows from equation (2) of definition 2.3.

The stack $D_{k}\left(\mathbb{P}^{N}, A, B, M\right)$ is visibly a closed substack of

$$
\bar{M}_{\left|\alpha^{(0)}\right|+r}\left(\mathbb{P}^{N}, d^{(0)}\right) \times_{\left(\mathbb{P}^{N}\right)^{r}} \prod_{i=1}^{r} \bar{M}_{\left|\alpha^{(i)}\right|+1}\left(\mathbb{P}^{N}, d^{(i)}\right),
$$

which in turn is a closed substack of $\bar{M}_{n}\left(\mathbb{P}^{N}, d\right)$ by BM] chapter 7 property III. To prove that it is contained in $\bar{M}_{\alpha}\left(\mathbb{P}^{N}, d\right)$ it suffices to show that a general element $\mathcal{C}=\left(C, x_{1}, \ldots, x_{n}, f\right) \in D_{k}\left(\mathbb{P}^{N}, A, B, M\right)$ satisfies the conditions of remark 1.4. As $\mathcal{C}$ is general, we have $C=C^{(0)} \cup \cdots \cup C^{(r)}$ where $C^{(0)} \in M_{r+\left|\alpha^{(0)}\right|}\left(H, d^{(0)}\right)$ and $C^{(i)} \in M_{\alpha^{(i)} \cup\left(m^{(i)}\right)}\left(\mathbb{P}^{N}, d^{(i)}\right)$. The condition of remark 1.4 is obvious for all connected components of $f^{-1}(H)$ besides $C^{(0)}$. As for $C^{(0)}$, the condition is exactly the " $\geq$ " part of equation (2) of definition 2.3.

Remark 2.5. We will see in proposition 4.4 that even for general $X$, the moduli spaces $D_{k}(X, A, B, M)$ satisfying equation (2) of definition 2.3 are proper substacks of $\bar{M}_{\alpha}(X, \beta)$ of expected codimension one. Thus we can view the virtual fundamental class of the $D_{k}(X, A, B, M)$ as well as of $D_{\alpha, k}(X, \beta)$ as cycles in the Chow group of $\bar{M}_{\alpha}(X, \beta)$ whose dimension is equal to the expected dimension of $\bar{M}_{\alpha}(X, \beta)$ minus one.

We can now state the main theorem of this paper.

Theorem 2.6. With notations as above, we have

$$
\left(\alpha_{k} \psi_{k}+e v_{k}^{*} Y\right) \cdot\left[\bar{M}_{\alpha}(X, \beta)\right]^{\text {virt }}=\left[\bar{M}_{\alpha+e_{k}}(X, \beta)\right]^{\text {virt }}+\left[D_{\alpha, k}(X, \beta)\right]^{\text {virt }}
$$

in the Chow group of $\bar{M}_{\alpha}(X, \beta)$, for all $1 \leq k \leq n$.

The proof will be given at the end of section 4 . 


\section{Proof OF THE MAIN THEOREM FOR HYPERPLANES IN $\mathbb{P}^{N}$}

In this section we will prove the main theorem 2.6 in the case where $X=\mathbb{P}^{N}$ and $Y=H$ is a hyperplane. Most of the proofs are generalized versions from those in [V], where the generalizations are quite straightforward. Recall that in construction 2.1 we defined a section $\sigma_{k}$ of a suitable line bundle on $\bar{M}_{\alpha}\left(\mathbb{P}^{N}, d\right)$ such that the zero locus of $\sigma_{k}$ has class $\alpha_{k} \psi_{k}+e v_{k}^{*} H$ and describes exactly those stable maps $\left(C, x_{1}, \ldots, x_{n}, f\right)$ where $f$ vanishes to order at least $\alpha_{k}+1$ along $H$ at $x_{k}$. For simplicity, we will restrict ourselves first to the case $\sum \alpha=d$ (note that the term $\left[\bar{M}_{\alpha+e_{k}}\left(\mathbb{P}^{N}, d\right)\right]^{\text {virt }}$ in the main theorem is then absent for degree reasons). We begin by proving a set-theoretic version of the main theorem.

Lemma 3.1. Assume that $\sum \alpha=d$. Then the zero locus of the section $\sigma_{k}$ on $\bar{M}_{\alpha}\left(\mathbb{P}^{N}, d\right)$ is equal to $D_{\alpha, k}\left(\mathbb{P}^{N}, d\right)$.

Proof. By construction, it is obvious that $\sigma_{k}$ vanishes on $D_{\alpha, k}\left(\mathbb{P}^{N}, d\right)$, so let us prove the converse. Let $\mathcal{C}=\left(C, x_{1}, \ldots, x_{n}, f\right) \in \bar{M}_{\alpha}\left(\mathbb{P}^{N}, d\right)$ be a stable map with $\sigma_{k}(\mathcal{C})=0$

Assume first that $x_{n}$ is an isolated point of $f^{-1}(H)$. As $f$ vanishes to order at least $\alpha_{k}+1$ along $H$ at $x_{k}$, this is a contradiction to remark 1.7 (i).

So $x_{n}$ is not an isolated point of $f^{-1}(H)$. Let $C^{(0)}$ be the connected component of $f^{-1}(H)$ containing $x_{k}$, and let $C^{(1)}, \ldots, C^{(r)}$ be the connected components of $\overline{C \backslash C^{(0)}}$. Let $m^{(i)}$ be the multiplicity of $\left.f\right|_{C^{(i)}}$ at $C^{(0)} \cap C^{(i)}$ along $H$, let $d^{(i)}$ be the degree of $f$ on $C^{(i)}$, and let $\alpha^{(i)}$ be the collection of the multiplicities $\alpha_{j}$ such that $x_{j} \in C^{(i)}$. Then it is obvious that $\mathcal{C} \in D_{k}\left(\mathbb{P}^{N}, A, B, M\right)$ with $A, B, M$ as in definition 2.2. Moreover, equation (2) of definition 2.3 is satisfied by remark 1.7 (ii) applied to $C^{(0)}$, hence it follows that $\mathcal{C} \in D_{\alpha, k}\left(\mathbb{P}^{N}, d\right)$.

Remark 3.2. As the spaces $D_{k}\left(\mathbb{P}^{N}, A, B, M\right)$ are irreducible and of codimension one by lemma 2.4, lemma 3.1 tells us that in the case $\sum \alpha=d$ we must have

$$
\left(\alpha_{k} \psi_{k}+e v_{k}^{*} H\right) \cdot\left[\bar{M}_{\alpha}\left(\mathbb{P}^{N}, d\right)\right]=\sum \lambda_{A, B, M}\left[D_{k}\left(\mathbb{P}^{N}, A, B, M\right)\right]^{\text {virt }}
$$

for some $\lambda_{A, B, M}$, where the sum is taken over all $A, B, M$ for which $D_{k}\left(\mathbb{P}^{N}, A, B, M\right)$ occurs in $D_{\alpha, k}\left(\mathbb{P}^{N}, d\right)$. Note that the virtual fundamental class of $D_{k}\left(\mathbb{P}^{N}, A, B, M\right)$ was defined to be $\frac{m^{(1)} \ldots m^{(r)}}{r !}$ times the usual one (where $r=|M|$ ), but that on the other hand every irreducible component of the zero locus of $\sigma_{k}$ (which is of the form $D_{k}\left(\mathbb{P}^{N}, A, B, M\right)$ for some $\left.A, B, M\right)$ gets counted $r$ ! times in the above sum, corresponding to the choice of order of the external components $C^{(1)}, \ldots, C^{(r)}$. Hence, to prove the main theorem for hyperplanes in $\mathbb{P}^{N}$ in the case $\sum \alpha=d$, we have to show that $\sigma_{k}$ vanishes along $D_{k}\left(\mathbb{P}^{N}, A, B, M\right)$ with multiplicity $m^{(1)} \cdots m^{(r)}$.

We will now prove the main theorem for $X=\mathbb{P}^{1}$ and $Y=H$ a point, in the case where $\sum \alpha=d$. The proof is very similar to the proof of $\mathbb{V}$ proposition 4.8 , in fact (modulo notations) identical up to the end where the section $\sigma_{k}$ comes into play, so we will only sketch these identical parts and refer to V] for details.

Proposition 3.3 (Main Theorem for $H \subset \mathbb{P}^{1}, \sum \alpha=d$ ). If $\sum \alpha=d$, then

$$
\left(\alpha_{k} \psi_{k}+e v_{k}^{*} H\right) \cdot\left[\bar{M}_{\alpha}\left(\mathbb{P}^{1}, d\right)\right]=\left[D_{\alpha, k}\left(\mathbb{P}^{1}, d\right)\right]^{v i r t}
$$

in the Chow group of $\bar{M}_{\alpha}\left(\mathbb{P}^{1}, d\right)$, for all $1 \leq k \leq n$. 
Proof. Let $D_{k}\left(\mathbb{P}^{1}, A, B, M\right)$ be a component of $D_{\alpha, k}\left(\mathbb{P}^{1}, d\right)$. By equation (2) of definition 2.3 we know that $\sum \alpha^{(0)}=\sum_{i} m^{(i)}$, call this number $d^{\prime}$. Moreover, we must obviously have $r>0$.

We start by defining two easier moduli spaces that model locally the situation at hand (in a sense that is made precise later). Fix a point $P \in \mathbb{P}^{1}$ distinct from $H$. Let $M \subset \bar{M}_{\left|\alpha^{(0)}\right|+r}\left(\mathbb{P}^{1}, d^{\prime}\right)$ be the closure of all degree- $d^{\prime}$ irreducible stable maps $\left(\mathbb{P}^{1},\left(x_{i}\right)_{1 \leq i \leq|\alpha(0)|},\left(y_{i}\right)_{1 \leq i \leq r}, f\right)$ such that

$$
f^{*} H=\sum_{i} \alpha_{i}^{(0)} x_{i} \quad \text { and } \quad f^{*} P=\sum_{i} m^{(i)} y_{i} .
$$

Let $D \subset \bar{M}_{\mid \alpha^{(0) \mid+r}}\left(\mathbb{P}^{1}, d^{\prime}\right)$ be the closure of all degree- $d^{\prime}$ reducible stable maps $\left(C^{(0)} \cup \cdots \cup C^{(r)},\left(x_{i}\right)_{1 \leq i \leq\left|\alpha^{(0)}\right|},\left(y_{i}\right)_{1 \leq i \leq r}, f\right)$ with $r+1$ components such that

- $f$ contracts $C^{(0)}$ to $H$, and $C^{(i)} \cap C^{(0)} \neq \emptyset$ for all $1 \leq i \leq r$,

- $x_{i} \in C^{(0)}$ for all $1 \leq i \leq\left|\alpha^{(0)}\right|$,

- $\left(\left.f\right|_{C^{(i)}}\right)^{*} H=m^{(i)}\left(C^{(i)} \cap C^{(0)}\right)$ and $\left(\left.f\right|_{C^{(i)}}\right)^{*} P=m^{(i)} y_{i}$ for all $1 \leq i \leq r$.

General elements of these moduli spaces look as follows (the picture represents the case $\alpha=(0,4,1)$ and $M=(2,3))$ :

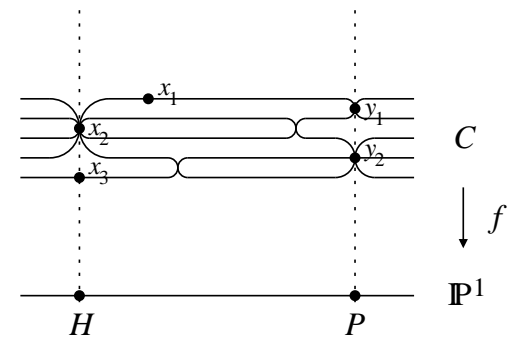

A general element in $M$

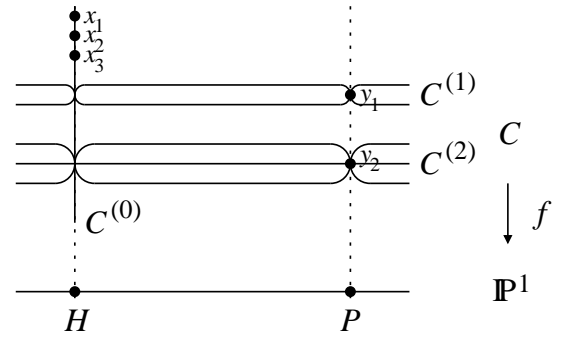

A general element in $D$

In short, in addition to our usual multiplicity requirements for $f^{*} H$ we require multiplicities $m^{(i)}$ over the point $P$ (so that the curves $C^{(i)}$ in $D$ are ramified completely over $H$ and $P$ for $i>0$ ).

We are now ready to compute the multiplicity of $\sigma_{k}$ to $D_{k}\left(\mathbb{P}^{1}, A, B, M\right)$ at a general element $\mathcal{C}^{\prime}=\left(C^{\prime}, x_{1}^{\prime}, \ldots, x_{n}^{\prime}, f^{\prime}\right)$. Let $\mathcal{C}=\left(C,\left(x_{i}\right),\left(y_{i}\right), f\right)$ be the unique stable map in $D$ whose internal component $C^{(0)}$ is equal to the internal component of $\mathcal{C}^{\prime}$, viewed as a marked curve whose marked points are the $x_{i}$ and the points $C^{(0)} \cap C^{(i)}$.

By construction, the stable maps $\mathcal{C}$ and $\mathcal{C}^{\prime}$ are étale locally isomorphic around $C^{(0)}$, so let $\left(U,\left(x_{i}\right),\left.f\right|_{U}\right)$ be a sufficiently small common étale neighborhood of $C^{(0)}$. By $\left[\mathrm{V}\right.$ proposition 4.3 the deformation spaces of $\mathcal{C}$ in $M$ and $\mathcal{C}^{\prime}$ in $\bar{M}_{\alpha}\left(\mathbb{P}^{1}, d\right)$ are products one of whose factors is the deformation space of $\left(U,\left(x_{i}\right),\left.f\right|_{U}\right)$, viewed as a map from $U$ to $\mathbb{P}^{1}$ satisfying the given multiplicity conditions at the points $x_{i}$. As the section $\sigma_{k}$ is defined on this common factor, the order of vanishing of $\sigma_{k}$ along $D_{k}\left(\mathbb{P}^{1}, A, B, M\right)$ in $\bar{M}_{\alpha}\left(\mathbb{P}^{1}, d\right)$ at the point $\mathcal{C}^{\prime}$ is equal to its order of vanishing along $D$ in $M$ at the point $\mathcal{C}$.

To simplify the calculations even further, we will now fix the marked curve $\left(C,\left(x_{i}\right),\left(y_{i}\right)\right)$. Consider the morphism $\pi: M \rightarrow \bar{M}_{|\alpha(0)|+r}$ given by forgetting the map $f$ and stabilizing if necessary. Note that $\pi$ will contract all external components of $\mathcal{C}$ as they only have two special points, so $\pi$ maps $\mathcal{C}$ to a general point of $\bar{M}_{|\alpha(0)|+r}$. 
Denote by $M^{\prime} \subset M$ and $D^{\prime} \subset D$ the fibers of this morphism over $\pi(\mathcal{C})$. Then the multiplicity we seek is equal to the multiplicity of $\sigma_{k}$ along $D^{\prime}$ in $M^{\prime}$ in the point $\mathcal{C}$.

But general elements in $M^{\prime}$ are actually easy to describe explicitly: choose $g_{1}, g_{2} \in \mathcal{O}_{\mathbb{P}^{1}}\left(d^{\prime}\right)$ with associated divisors

$$
\left(g_{1}\right)=\sum_{i} \alpha_{i}^{(0)} x_{i} \quad \text { and } \quad\left(g_{2}\right)=\sum_{i} m^{(i)} y_{i}
$$

where $x_{i}$ and $y_{i}$ are now fixed points in $\mathbb{P}^{1}$, determined by the element $\pi(\mathcal{C}) \in$ $\bar{M}_{|\alpha(0)|+r}$. Then a general stable map in $M^{\prime}$ is of the form

$$
\mathcal{C}_{\lambda}=\left(\mathbb{P}^{1},\left(x_{i}\right),\left(y_{i}\right), f\right) \quad \text { where } \quad f: \mathbb{P}^{1} \rightarrow \mathbb{P}^{1}, x \mapsto\left(\lambda g_{1}(x): g_{2}(x)\right)
$$

for $\lambda \in \mathbb{C}^{*}$. (Here we have chosen coordinates on the target $\mathbb{P}^{1}$ such that $H=(0: 1)$ and $P=(1: 0)$.) The locus $D^{\prime} \subset M^{\prime}$, which is set-theoretically the zero locus of $\sigma_{k}$, corresponds to the degeneration $\lambda \rightarrow 0$.

After a finite base change we can extend the family $\left\{\mathcal{C}_{\lambda}\right\}$ to $\lambda=0$. The central fiber $\mathcal{C}_{0}$ of this extended family is equal to $\mathcal{C}$.

Let $z$ be a local coordinate around $x_{k} \in \mathbb{P}^{1}$. This means that $z$ is a local coordinate around $x_{k}$ on all $\mathcal{C}_{\lambda}$ with $\lambda \neq 0$, and in fact it extends to a local coordinate around $x_{k}$ for $\lambda=0$. Consider the local trivialization of the line bundle $L_{k}^{\otimes \alpha_{k}} \otimes e v_{k}^{*} \mathcal{O}(H)$ given by $d z\left(x_{k}\right)^{\otimes \alpha_{k}} \otimes h\left(x_{k}\right) \mapsto 1$ (where $h \in H^{0}\left(\mathbb{P}^{1}, \mathcal{O}(H)\right.$ ) is the section vanishing at $H$ that is used to define $\sigma_{k}$ ). Then by construction, the section $\sigma_{k}$ on the family $\mathcal{C}_{\lambda}$ is given by $\left.\lambda \mapsto \frac{\partial^{\alpha} k}{\partial z^{\alpha} k} \lambda g_{1}(z)\right|_{z=x_{k}}$ in this local trivialization. In particular, this has a zero of first order in $\lambda$ at $\lambda=0$. This means that the class of the zero locus of $\sigma_{k}$ on $M^{\prime}$ is

$$
\left(\alpha_{k} \psi_{k}+e v_{k}^{*} H\right) \cdot\left[M^{\prime}\right]=1 \cdot\left[\mathcal{C}_{\lambda}\right]
$$

for general $\lambda$.

Finally, as the automorphism group of a general $\mathcal{C}_{\lambda}$ is trivial, whereas the automorphism group of $\mathcal{C}$ is $\mathbb{Z}_{m^{(1)}} \times \cdots \times \mathbb{Z}_{m^{(r)}}$, we conclude that

$$
\left(\alpha_{k} \psi_{k}+e v_{k}^{*} H\right) \cdot\left[M^{\prime}\right]=m^{(1)} \cdots m^{(r)} \cdot[\mathcal{C}] .
$$

Hence the statement of the proposition follows from remark 3.2 .

Corollary 3.4 (Main Theorem for $H \subset \mathbb{P}^{N}, \sum \alpha=d$ ). If $\sum \alpha=d$, then

$$
\left(\alpha_{k} \psi_{k}+e v_{k}^{*} H\right) \cdot\left[\bar{M}_{\alpha}\left(\mathbb{P}^{N}, d\right)\right]=\left[D_{\alpha, k}\left(\mathbb{P}^{N}, d\right)\right]^{\text {virt }}
$$

in the Chow group of $\bar{M}_{\alpha}\left(\mathbb{P}^{N}, d\right)$, for all $1 \leq k \leq n$.

Proof. (Compare to $[\mathrm{V}]$ theorem 6.1.) By the previous proposition we can assume that $N \geq 2$. Consider a general element $\mathcal{C}=\left(C, x_{1}, \ldots, x_{n}, f\right)$ of a component $D_{k}\left(\mathbb{P}^{N}, A, B, M\right)$ of $D_{\alpha, k}\left(\mathbb{P}^{N}, d\right)$. Let $A \subset H$ be a general $(N-2)$-plane. The projection from $A$ in $\mathbb{P}^{N}$ induces a rational map $\rho_{A}: \bar{M}_{n}\left(\mathbb{P}^{N}, d\right) \rightarrow \bar{M}_{n}\left(\mathbb{P}^{1}, d\right)$. By V7 proposition 5.5 the map $\rho_{A}$ is defined and smooth at $\mathcal{C}$. Moreover, $\rho_{A}$ maps $D_{k}\left(\mathbb{P}^{N}, A, B, M\right)$ to $D_{k}\left(\mathbb{P}^{1}, A, B, M\right)$ at the points of $D_{k}\left(\mathbb{P}^{N}, A, B, M\right)$ where it is defined, and the section $\sigma_{k}$ on $\bar{M}_{\alpha}\left(\mathbb{P}^{1}, d\right)$ pulls back along $\rho_{A}$ to the section $\sigma_{k}$ on $\bar{M}_{\alpha}\left(\mathbb{P}^{N}, d\right)$. Hence the multiplicity of $\sigma_{k}$ on $\bar{M}_{\alpha}\left(\mathbb{P}^{N}, d\right)$ along $D_{k}\left(\mathbb{P}^{N}, A, B, M\right)$ is the same as the multiplicity of $\sigma_{k}$ on $\bar{M}_{\alpha}\left(\mathbb{P}^{1}, d\right)$ along $D_{k}\left(\mathbb{P}^{1}, A, B, M\right)$. The corollary then follows from proposition 3.3 and remark 3.2 . 
Corollary 3.5 (Main Theorem for $H \subset \mathbb{P}^{N}$ ). We have

$$
\left(\alpha_{k} \psi_{k}+e v_{k}^{*} H\right) \cdot\left[\bar{M}_{\alpha}\left(\mathbb{P}^{N}, d\right)\right]=\left[\bar{M}_{\alpha+e_{k}}\left(\mathbb{P}^{N}, d\right)\right]+\left[D_{\alpha, k}\left(\mathbb{P}^{N}, d\right)\right]^{v i r t}
$$

in the Chow group of $\bar{M}_{\alpha}\left(\mathbb{P}^{N}, d\right)$, for all $1 \leq k \leq n$.

Proof. Let $s=d-\sum \alpha$, and let $\alpha^{\prime}=\alpha \cup(1, \ldots, 1)$ such that $\sum \alpha^{\prime}=d$. By corollary 3.4 we know that

$$
\left(\alpha_{k}^{\prime} \psi_{k}^{\prime}+e v_{k}^{\prime *} H\right) \cdot\left[\bar{M}_{\alpha^{\prime}}\left(\mathbb{P}^{N}, d\right)\right]=\left[D_{\alpha^{\prime}, k}\left(\mathbb{P}^{N}, d\right)\right]^{v i r t}
$$

for $1 \leq k \leq n$, where $\psi_{k}^{\prime}$ is the $k$-th cotangent line class on $\bar{M}_{n+s}\left(\mathbb{P}^{N}, d\right)$, and $e v_{k}^{\prime}$ the evaluation map $\bar{M}_{n+s}\left(\mathbb{P}^{N}, d\right) \rightarrow \mathbb{P}^{N}$ at the $k$-th marked point. We will show that the push-forward of this equation along the morphism $\phi: \bar{M}_{\alpha^{\prime}}\left(\mathbb{P}^{N}, d\right) \rightarrow \bar{M}_{\alpha}\left(\mathbb{P}^{N}, d\right)$ that forgets the additional $s$ marked points is exactly the statement of the corollary.

First note that $\alpha_{k}^{\prime}=\alpha_{k}$ and $e v_{k}^{\prime}=e v_{k} \circ \phi$. For the computation of the pushforward of $\psi_{k}^{\prime}$ we may assume that $\alpha_{k}>0$, as otherwise there is no $\psi_{k}^{\prime}$-term in (3). It is well-known that $\psi_{k}^{\prime}=\phi^{*} \psi_{k}+\gamma$, where the correction term $\gamma$ is the class of the locus of those stable maps $\mathcal{C}=\left(C, x_{1}, \ldots, x_{n+s}, f\right)$ where $\phi$ contracts the irreducible component $Z$ of $C$ on which $x_{k}$ lies, i.e. where $Z$ is an unstable component of the prestable map $\left(C, x_{1}, \ldots, x_{n}, f\right)$. This can only happen if $Z$ is contracted by $f$, in particular $\sigma_{k}(\mathcal{C})=0$, so by lemma 3.1 the cycle $\gamma$ must be a union of some of the components of $D_{k}\left(\mathbb{P}^{N}, A, B, M\right)$ of $D_{\alpha^{\prime}, k}\left(\mathbb{P}^{N}, d\right)$. To determine which of them occur in $\gamma$, we can assume that $\mathcal{C}$ is a generic element of some $D_{k}\left(\mathbb{P}^{N}, A, B, M\right)$. It is easy to see that $\phi$ contracts $Z=C^{(0)}$ if and only if $r=|M|=1, d^{(0)}=0$, and the marked points on $Z$ are $x_{k}$ and at least one of the points $x_{n+1}, \ldots, x_{n+s}$. If there is more than one of these points on $Z$, the map $\phi$ has positive-dimensional fibers on $D_{k}\left(\mathbb{P}^{N}, A, B, M\right)$, and hence $\phi_{*}\left[D_{k}\left(\mathbb{P}^{N}, A, B, M\right)\right]$ vanishes, hence we can assume that the marked points on $Z$ are exactly $x_{k}$ and one of the forgotten points. Then $\phi(\mathcal{C})$ contracts $Z$, so by remark 1.7 the stable map $\phi(\mathcal{C})$ will be irreducible with multiplicity $\alpha_{k}+1$ at $x_{k}$ to $H$. This means that $\phi\left(D_{k}\left(\mathbb{P}^{N}, A, B, M\right)\right)=\bar{M}_{\alpha+e_{k}}\left(\mathbb{P}^{N}, d\right)$. As there is an $s !$-fold choice of order of the forgotten marked points, we have shown that

$$
\phi_{*} \gamma \cdot\left[M_{\alpha^{\prime}}\left(\mathbb{P}^{N}, d\right)\right]=s ! \cdot\left[\bar{M}_{\alpha+e_{k}}\left(\mathbb{P}^{N}, d\right)\right]
$$

and that therefore the left hand side of the push-forward of (3) by $\phi$ is equal to

$$
s ! \cdot\left(\alpha_{k} \psi_{k}+e v_{k}^{*} H\right) \cdot\left[\bar{M}_{\alpha}\left(\mathbb{P}^{N}, d\right)\right]+\alpha_{k} s ! \cdot\left[\bar{M}_{\alpha+e_{k}}\left(\mathbb{P}^{N}, d\right)\right] .
$$

Now we look at the right hand side of the push-forward of (3) by $\phi$. Consider a component $D_{k}\left(\mathbb{P}^{N}, A, B, M\right)$ of $D_{\alpha^{\prime}, k}\left(\mathbb{P}^{N}, d\right)$ and let $\mathcal{C}=\left(C, x_{1}, \ldots, x_{n+s}, f\right)$ be a generic element of this component. For the push-forward of this component by $\phi$ to be non-zero, the fibers of $\phi$ have to be zero-dimensional, i.e. there must not be a deformation of $\mathcal{C}$ inside $D_{k}\left(\mathbb{P}^{N}, A, B, M\right)$ that changes nothing but the position of the points $x_{n+1}, \ldots, x_{n+s}$. In particular this means that we must have one of the following two cases:

- $C^{(0)}$ contains none of the points $x_{n+1}, \ldots, x_{n+s}$, i.e. the points $x_{n+1}, \ldots, x_{n+s}$ are just the $s$ unmarked transverse points of intersection of $\phi(\mathcal{C})$ with $H$. In this case, the map $\phi$ does not contract any components of $C$, and it changes no multiplicities to $H$. Hence, the push-forward by $\phi$ of all these components together is just $s ! \cdot\left[D_{\alpha, k}\left(\mathbb{P}^{N}, d\right)\right]^{\text {virt }}$. 
- $C^{(0)}$ is a contracted component, i.e. $d^{(0)}=0, r=|M|=1$, and the marked points on $C^{(0)}$ are exactly $x_{k}$ and one of the points $x_{n+1}, \ldots, x_{n+s}$. As above, the push-forward of such a component yields $\bar{M}_{\alpha+e_{k}}\left(\mathbb{P}^{N}, d\right)$, and it occurs with multiplicity $\left(\alpha_{k}+1\right) s$ !, where the factor $\alpha_{k}+1$ comes from the definition of the virtual fundamental class of $D_{k}\left(\mathbb{P}^{N}, A, B, M\right)$.

Put together, we have shown that the push-forward of the right hand side of (3) by $\phi$ is equal to

$$
s ! \cdot\left[D_{\alpha, k}\left(\mathbb{P}^{N}, d\right)\right]^{v i r t}+\left(\alpha_{k}+1\right) s ! \cdot\left[\bar{M}_{\alpha+e_{k}}\left(\mathbb{P}^{N}, d\right)\right] .
$$

Combining this with (4), we get the desired result.

\section{Proof of the MAIN THEOREM FOR VERY AMPLE HYPERSURFACES}

Let $X$ be a smooth complex projective variety and $Y$ a smooth very ample hypersurface. We fix the following notation. Let $i: Y \rightarrow X$ be the inclusion map. For $\beta \in H_{2}^{+}(X)$ we denote by $\bar{M}_{n}(Y, \beta)$ the disjoint union of all moduli spaces $\bar{M}_{n}\left(Y, \beta^{\prime}\right)$ for $\beta^{\prime} \in H_{2}^{+}(Y)$ such that $i_{*} \beta^{\prime}=\beta$. Consider the embedding $\varphi: X \rightarrow \mathbb{P}^{N}$ given by the complete linear system $|Y|$ and let $H \subset \mathbb{P}^{N}$ be the hyperplane such that $\varphi^{-1}(H)=Y$. There is an induced morphism $\phi: \bar{M}_{n}(X, \beta) \rightarrow \bar{M}_{n}\left(\mathbb{P}^{N}, d\right)$, where $d=Y \cdot \beta$. In this section we will show that the "pull-back" of the main theorem for $H \subset \mathbb{P}^{N}$ by $\phi$ yields the main theorem for $Y \subset X$. The most difficult part of the proof is to show that the spaces $D_{\alpha, k}\left(\mathbb{P}^{N}, d\right)$ pull back to $D_{\alpha, k}(X, \beta)$ (proposition 4.4). Recall that curves in $D_{\alpha, k}(X, \beta)$ are reducible curves with one component in $Y$ (and some multiplicity conditions). Hence we will show first that the moduli spaces of curves in $Y$ (lemma 4.2) and those of reducible curves in $X$ (lemma 4.3) pull back nicely under $\phi$.

Convention 4.1. In this section, all occurring spaces are equipped with virtual fundamental classes as follows.

- The moduli spaces of stable maps $\bar{M}_{n}(\cdot, \cdot)$ have virtual fundamental classes constructed e.g. in [B], BF].

- The moduli spaces $M_{\alpha}(\cdot, \cdot), D_{k}(\ldots)$, and $D_{\alpha, k}(\ldots)$ have virtual fundamental classes constructed in definitions 1.18, 2.2, and 2.3, respectively.

- The varieties $Y, X, H$, and $\mathbb{P}^{N}$ are equipped with their usual fundamental class.

- The virtual fundamental class of a disjoint union of spaces is the sum of the virtual fundamental classes of its components.

- In any fiber product $V_{1} \times_{V} V_{2}$ occurring in this section, $V$ will always be smooth and equipped with the usual fundamental class. The virtual fundamental class of the fiber product is then taken to be the one induced by the virtual fundamental classes of $V_{1}$ and $V_{2}$ in the sense of remark 1.19.

When we say that two spaces $V_{1}$ and $V_{2}$ are equal we will always mean that $V_{1}$ and $V_{2}$ are isomorphic and that $\left[V_{1}\right]^{\text {virt }}=\left[V_{2}\right]^{\text {virt }}$ under this isomorphism. We will write this as $V_{1} \equiv V_{2}$.

Lemma 4.2. For any $n \geq 0$ and $\beta \in H_{2}^{+}(X)$ we have

$$
\bar{M}_{n}(Y, \beta) \equiv \bar{M}_{n}(H, d) \times_{\bar{M}_{n}\left(\mathbb{P}^{N}, d\right)} \bar{M}_{n}(X, \beta) .
$$


Proof. As $Y=H \cap X \subset \mathbb{P}^{N}$, it follows from the definitions that the diagram of inclusions

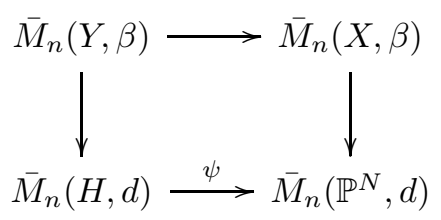

is cartesian. We denote by $\pi_{X}: \bar{M}_{n+1}(X, \beta) \rightarrow \bar{M}_{n}(X, \beta)$ the universal curve and by $f_{X}: \bar{M}_{n+1}(X, \beta) \rightarrow X$ its evaluation map, and similarly for the moduli spaces of maps to $Y, H$, and $\mathbb{P}^{N}$. Applying the functor $R \pi_{Y *} f_{Y}^{*}$ to the distinguished triangle

$$
\left.\left.L_{X}\right|_{Y} \rightarrow L_{Y} \rightarrow L_{Y / X} \rightarrow L_{X}\right|_{Y}[1]
$$

on $Y$, we get the distinguished triangle

$$
\begin{aligned}
& \left.\left.R \pi_{Y *}\left(f_{X}^{*} L_{X}\right)\right|_{\bar{M}_{n+1}(Y, \beta)} \rightarrow R \pi_{Y *} f_{Y}^{*} L_{Y} \rightarrow R \pi_{Y *}\left(f_{H}^{*} L_{H / \mathbb{P}^{N}}\right)\right|_{\bar{M}_{n+1}(Y, \beta)} \\
& \left.\quad \rightarrow R \pi_{Y *}\left(f_{X}^{*} L_{X}\right)\right|_{\bar{M}_{n+1}(Y, \beta)}[1]
\end{aligned}
$$

on $\bar{M}_{n}(Y, \beta)$. By [B] proposition 5 , the vector bundle $f_{X}^{*} L_{X}$ is quasi-isomorphic to a complex $K$ of vector bundles on $\bar{M}_{n+1}(X, \beta)$ such that $R \pi_{X *} K$ is also a complex of vector bundles. As $\pi_{X}$ is flat, it follows from the theorem on cohomology and base change that $\left(R \pi_{X *} K\right)_{\bar{M}_{n}(Y, \beta)}=R \pi_{Y *}\left(\left.K\right|_{\bar{M}_{n+1}(Y, \beta)}\right)$. The same argument applies to $f_{H}^{*} L_{H / \mathbb{P}^{N}}$ instead of $f_{X}^{*} L_{X}$, so we arrive at the distinguished triangle

$$
\begin{aligned}
& \left.\left.\left(R \pi_{X *} f_{X}^{*} L_{X}\right)\right|_{\bar{M}_{n}(Y, \beta)} \rightarrow R \pi_{Y *} f_{Y}^{*} L_{Y} \rightarrow\left(R \pi_{H *} f_{H}^{*} L_{H / \mathbb{P}^{N}}\right)\right|_{\bar{M}_{n}(Y, \beta)} \\
& \left.\quad \rightarrow\left(R \pi_{X *} f_{X}^{*} L_{X}\right)\right|_{\bar{M}_{n}(Y, \beta)}[1] .
\end{aligned}
$$

Starting with the distinguished triangle of $L_{H / \mathbb{P}^{N}}$ instead of $L_{Y / X}$ in (6), the same calculation as above shows that we also have a distinguished triangle on $\bar{M}_{n}(H, d)$

$$
\begin{aligned}
& \left.\left(R \pi_{\mathbb{P}^{N} *} f_{\mathbb{P}^{N}}^{*} L_{\mathbb{P}^{N}}\right)\right|_{\bar{M}_{n}(H, d)} \rightarrow R \pi_{H *} f_{H}^{*} L_{H} \rightarrow R \pi_{H *} f_{H}^{*} L_{H / \mathbb{P}^{N}} \\
& \left.\quad \rightarrow\left(R \pi_{\mathbb{P}^{N} *} f_{\mathbb{P}^{N}}^{*} L_{\mathbb{P}^{N}}\right)\right|_{\bar{M}_{n}(H, d)}[1] .
\end{aligned}
$$

But the first and second term in this sequence are just $\left.L_{\bar{M}_{n}\left(\mathbb{P}^{N}, d\right) / \mathcal{M}_{n}}\right|_{\bar{M}_{n}(H, d)}$ and $L_{\bar{M}_{n}(H, d) / \mathcal{M}_{n}}$, where $\mathcal{M}_{n}$ denotes the stack of prestable $n$-pointed rational curves. Hence we see that $R \pi_{H *} f_{H}^{*} L_{H / \mathbb{P}^{N}}=L_{\bar{M}_{n}(H, d) / \bar{M}_{n}\left(\mathbb{P}^{N}, d\right)}$. So (7) becomes

$$
\begin{aligned}
& \left.\left.\left(R \pi_{X *} f_{X}^{*} L_{X}\right)\right|_{\bar{M}_{n}(Y, \beta)} \rightarrow R \pi_{Y *} f_{Y}^{*} L_{Y} \rightarrow L_{\bar{M}_{n}(H, d) / \bar{M}_{n}\left(\mathbb{P}^{N}, d\right)}\right|_{\bar{M}_{n}(Y, \beta)} \\
& \left.\quad \rightarrow\left(R \pi_{X *} f_{X}^{*} L_{X}\right)\right|_{\bar{M}_{n}(Y, \beta)}[1] .
\end{aligned}
$$

As the first two terms in this sequence are the relative obstruction theories of $\bar{M}_{n}(X, \beta)$ and $\bar{M}_{n}(Y, \beta)$ over $\mathcal{M}_{n}$, respectively, we get a homomorphism of this distinguished triangle to

$$
\begin{aligned}
& \left.L_{\bar{M}_{n}(X, \beta) / \mathcal{M}_{n}}\right|_{\bar{M}_{n}(Y, \beta)} \rightarrow L_{\bar{M}_{n}(Y, \beta) / \mathcal{M}_{n}} \rightarrow L_{\bar{M}_{n}(Y, \beta) / \bar{M}_{n}(X, \beta)} \\
& \left.\quad \rightarrow L_{\bar{M}_{n}(X, \beta) / \mathcal{M}_{n}}\right|_{\bar{M}_{n}(Y, \beta)}[1] .
\end{aligned}
$$

Hence, by $\left[\mathrm{BF}\right.$ proposition 7.5 it follows that $\psi^{!}\left[\bar{M}_{n}(X, \beta)\right]^{\text {virt }}=\left[\bar{M}_{n}(Y, \beta)\right]^{\text {virt }}$ in (5). This proves the lemma. 
Lemma 4.3. Let $n^{(i)} \geq 0$ and $d^{(i)} \geq 0$ such that $\sum_{i} n^{(i)}=n$ and $\sum_{i} d^{(i)}=d$. Then

$$
\begin{aligned}
& \coprod_{\left(\beta^{(i)}\right)}\left(\bar{M}_{n^{(0)}+r}\left(X, \beta^{(0)}\right) \times_{X^{r}} \prod_{i=1}^{r} \bar{M}_{n^{(i)}+1}\left(X, \beta^{(i)}\right)\right) \equiv \\
& \quad\left(\bar{M}_{n^{(0)}+r}\left(\mathbb{P}^{N}, d^{(0)}\right) \times_{\left(\mathbb{P}^{N}\right)^{r}} \prod_{i=1}^{r} \bar{M}_{n^{(i)}+1}\left(\mathbb{P}^{N}, d^{(i)}\right)\right) \times_{\bar{M}_{n}\left(\mathbb{P}^{N}, d\right)} \bar{M}_{n}(X, \beta),
\end{aligned}
$$

where the union is taken over all $\left(\beta^{(i)}\right)$ with $Y \cdot \beta^{(i)}=d^{(i)}$ for all $i$, and where the maps to $X^{r}$ and $\left(\mathbb{P}^{N}\right)^{r}$ are given in the same way as in definition 2.8.

Proof. In the language of $\mathrm{BM}$, let $\tau$ be the graph corresponding to rational curves with components $C^{(0)}, \ldots, C^{(r)}$ such that $C^{(0)} \cap C^{(i)} \neq \emptyset$ for all $i>0$ and $C^{(i)}$ has $n^{(i)}$ marked points for $i \geq 0$. Let $\mathcal{M}_{n}$ be the stack of prestable $n$-pointed rational curves, and let $\mathcal{M}_{\tau} \subset \mathcal{M}_{n}$ be the substack of $\tau$-marked prestable curves, as defined in [BM definition 2.6. Moreover, we will abbreviate the moduli spaces in the large brackets in the statement of the lemma as $\bar{M}_{\tau}\left(X,\left(\beta^{(i)}\right)\right)$ and $\bar{M}_{\tau}\left(\mathbb{P}^{N},\left(d^{(i)}\right)\right)$, respectively.

Consider the commutative diagram

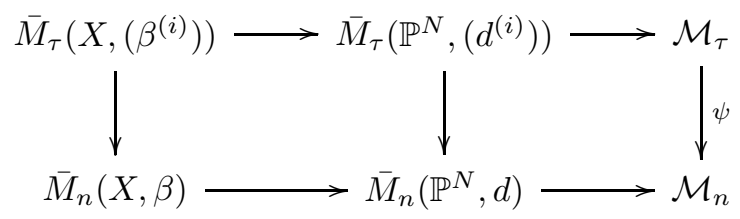

where none of the maps involves stabilization of the underlying prestable curves. By B lemma 10, the right square and the big square are cartesian, so the left one is also cartesian. Moreover, by the same lemma, $\psi^{!}\left[\bar{M}_{n}(X, \beta)\right]^{\text {virt }}=\left[\bar{M}_{\tau}\left(X,\left(\beta^{(i)}\right)\right)\right]^{\text {virt }}$.

Proposition 4.4. For any $1 \leq k \leq n$ we have

$$
D_{\alpha, k}(X, \beta) \equiv D_{\alpha, k}\left(\mathbb{P}^{N}, d\right) \times_{\bar{M}_{n}\left(\mathbb{P}^{N}, d\right)} \bar{M}_{n}(X, \beta)
$$

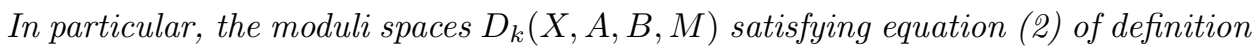
2.3 are proper substacks of $\bar{M}_{\alpha}(X, \beta)$ of expected codimension one.

Proof. We consider a component $D_{k}\left(\mathbb{P}^{N}, A,\left(d^{(i)}\right), M\right)$ of $D_{\alpha, k}\left(\mathbb{P}^{N}, d\right)$ and show that the fiber product of this component with $\bar{M}_{n}(X, \beta)$ over $\bar{M}_{n}\left(\mathbb{P}^{N}, d\right)$ is the union of all $D_{k}\left(X, A,\left(\beta^{(i)}\right), M\right)$ such that $Y \cdot \beta^{(i)}=d^{(i)}$.

We start with the pull-back compatibility statement for general curves of the form $C^{(0)} \cup \cdots \cup C^{(r)}$ with $C^{(0)} \cap C^{(i)} \neq \emptyset$, as given in lemma 4.3. Taking the fiber product of this equation with $\bar{M}_{n^{(0)}+r}\left(H, d^{(0)}\right)$ over $\bar{M}_{n^{(0)}+r}\left(\mathbb{P}^{N}, d^{(0)}\right)$ (i.e. requiring the central component $C^{(0)}$ to lie in $H$ ) and using lemma 4.2 on the left hand side yields

$$
\begin{aligned}
& \coprod_{\left(\beta^{(i)}\right)}\left(\bar{M}_{n^{(0)}+r}\left(Y, \beta^{(0)}\right) \times_{X} \prod_{i=1}^{r} \bar{M}_{n^{(i)}+1}\left(X, \beta^{(i)}\right)\right) \equiv \\
& \quad\left(\bar{M}_{n^{(0)}+r}\left(H, d^{(0)}\right) \times_{\left(\mathbb{P}^{N}\right)^{r}} \prod_{i=1}^{r} \bar{M}_{n^{(i)}+1}\left(\mathbb{P}^{N}, d^{(i)}\right)\right) \times_{\bar{M}_{n}\left(\mathbb{P}^{N}, d\right)} \bar{M}_{n}(X, \beta) .
\end{aligned}
$$


This can obviously be written in a more complicated way as

$$
\begin{aligned}
& \coprod_{\left(\beta^{(i)}\right)}\left(\bar{M}_{n^{(0)}+r}\left(Y, \beta^{(0)}\right) \times_{Y^{r}}\left(H^{r} \times_{\left(\mathbb{P}^{N}\right)^{r}} \prod_{i=1}^{r} \bar{M}_{n^{(i)}+1}\left(X, \beta^{(i)}\right)\right)\right) \\
& \quad\left(\bar{M}_{n^{(0)}+r}\left(H, d^{(0)}\right) \times_{H^{r}}\left(H^{r} \times_{\left(\mathbb{P}^{N}\right)^{r}} \prod_{i=1}^{r} \bar{M}_{n^{(i)}+1}\left(\mathbb{P}^{N}, d^{(i)}\right)\right)\right) \times_{\bar{M}_{n}\left(\mathbb{P}^{N}, d\right)} \bar{M}_{n}(X, \beta) .
\end{aligned}
$$

Note that $H \times_{\mathbb{P}^{N}} \bar{M}_{n^{(i)}+1}\left(\mathbb{P}^{N}, d^{(i)}\right) \equiv \bar{M}_{\tilde{\alpha}^{(i)}}\left(\mathbb{P}^{N}, d^{(i)}\right)$ for all $i>0$, where $\tilde{\alpha}^{(i)}=$ $(0, \ldots, 0,1)$. So we get

$$
\begin{aligned}
& \coprod_{\left(\beta^{(i)}\right)}\left(\bar{M}_{n^{(0)}+r}\left(Y, \beta^{(0)}\right) \times_{Y^{r}} \prod_{i=1}^{r} \bar{M}_{\tilde{\alpha}^{(i)}}\left(\mathbb{P}^{N}, d^{(i)}\right) \times_{\bar{M}_{n^{(i)}+1}\left(\mathbb{P}^{N}, d^{(i)}\right)} \bar{M}_{n^{(i)}+1}\left(X, \beta^{(i)}\right)\right) \equiv \\
& \left(\bar{M}_{n^{(0)}+r}\left(H, d^{(0)}\right) \times_{H^{r}} \prod_{i=1}^{r} \bar{M}_{\tilde{\alpha}^{(i)}}\left(\mathbb{P}^{N}, d^{(i)}\right)\right) \times_{\bar{M}_{n}\left(\mathbb{P}^{N}, d\right)} \bar{M}_{n}(X, \beta) .
\end{aligned}
$$

Finally, we take the fiber product of this equation with $\bar{M}_{\alpha^{(i)} \cup\left(m^{(i)}\right)}\left(\mathbb{P}^{N}, d\right)$ over $\bar{M}_{\tilde{\alpha}^{(i)}}\left(\mathbb{P}^{N}, d\right)$ for all $i>0$, yielding the same equation with the $\tilde{\alpha}^{(i)}$ replaced by $\alpha^{(i)} \cup$ $\left(m^{(i)}\right)$. By definition, this is then exactly the equation stated in the proposition.

We are now ready to give the proof of our main theorem.

Proof (of theorem 2.0). Consider the cartesian diagram

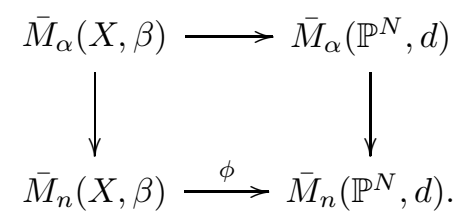

The main theorem for $H \subset \mathbb{P}^{N}$ (see corollary 3.5) gives an equation in the Chow group of $\bar{M}_{\alpha}\left(\mathbb{P}^{N}, d\right)$. We pull this equation back by $\phi$ to get an equation in the Chow group of $\bar{M}_{\alpha}(X, \beta)$. As the morphism $\phi$ does not involve any contractions of the underlying prestable curves, the cotangent line class $\psi_{k}$ on $\bar{M}_{n}\left(\mathbb{P}^{N}, d\right)$ pulls back to the cotangent line class $\psi_{k}$ on $\bar{M}_{n}(X, \beta)$. So by definition the left hand side of corollary 3.5 pulls back to $\left(\alpha_{k} \psi_{k}+e v^{*} Y\right) \cdot\left[\bar{M}_{\alpha}(X, \beta)\right]^{v i r t}$. In the same way, $\left[\bar{M}_{\alpha+e_{k}}\left(\mathbb{P}^{N}, d\right)\right]$ pulls back to $\left[\bar{M}_{\alpha+e_{k}}(X, \beta)\right]^{v i r t}$. Finally, proposition 4.4 shows that $\left[D_{\alpha, k}\left(\mathbb{P}^{N}, d\right)\right]^{\text {virt }}$ pulls back to $\left[D_{\alpha, k}(X, \beta)\right]^{\text {virt }}$.

Remark 4.5. We expect that the statement of the main theorem 2.6 is true even under weaker assumptions on the hypersurface $Y$. For example, if $Y$ is not very ample but the complete linear system $|Y|$ on $X$ is base-point free, we still get a morphism $X \rightarrow \mathbb{P}^{N}$ defined by $|Y|$. The definition of the moduli spaces of relative invariants essentially carries over without change to this case. The main (but probably little) problem is that the morphism $\phi$ in the cartesian diagram of definition 1.18 now may involve stabilization of the underlying prestable curves. This makes many points in the arguments of this paper more subtle, but we expect that a version of the main theorem can be proven also in this case. 


\section{ENUMERATIVE APPLICATIONS}

As usual, the first thing to do to get enumerative results from moduli spaces of maps is to define invariants by intersecting the virtual fundamental class of the moduli space with various cotangent line classes and pull-backs of classes via evaluation maps. Note that from the spaces $\bar{M}_{\alpha}(X, \beta)$ we always have evaluation maps $e v_{k}$ to $X$ for $1 \leq k \leq|\alpha|$, and in addition evaluation maps $\tilde{e v}_{k}$ to $Y$ for all $k$ with $\alpha_{k}>0$.

Definition 5.1. Let $\beta \in H_{2}^{+}(X), n \geq 0, k_{1}, \ldots, k_{n} \geq 0$, and $\gamma_{1}, \ldots, \gamma_{n} \in A^{*}(X)$. Then the restricted Gromov-Witten invariants of $Y$ are defined as

$$
I_{n, \beta}^{Y}\left(\gamma_{1} \psi^{k_{1}}, \ldots, \gamma_{n} \psi^{k_{n}}\right)=e v_{1}^{*} \gamma_{1} \cdot \psi_{1}^{k_{1}} \ldots e v_{n}^{*} \gamma_{n} \cdot \psi_{n}^{k_{n}} \cdot\left[\bar{M}_{n}(Y, \beta)\right]^{\text {virt }} \in \mathbb{Q}
$$

if $\sum_{i}\left(\operatorname{codim} \gamma_{i}+k_{i}\right)=\operatorname{vdim} \bar{M}_{n}(Y, \beta)$. Similarly, for any $\alpha=\left(\alpha_{1}, \ldots, \alpha_{n}\right)$ the restricted relative Gromov-Witten invariants of $Y \subset X$ are defined as

$$
I_{\alpha, \beta}\left(\gamma_{1} \psi^{k_{1}}, \ldots, \gamma_{n} \psi^{k_{n}}\right)=e v_{1}^{*} \gamma_{1} \cdot \psi_{1}^{k_{1}} \cdots e v_{n}^{*} \gamma_{n} \cdot \psi_{n}^{k_{n}} \cdot\left[\bar{M}_{\alpha}(X, \beta)\right]^{v i r t} \in \mathbb{Q}
$$

if $\sum_{i}\left(\operatorname{codim} \gamma_{i}+k_{i}\right)=\operatorname{vdim} \bar{M}_{\alpha}(X, \beta)$. This definition can obviously be generalized in the following two ways:

(i) We can take cohomology classes $\tilde{\gamma}_{k} \in A^{*}(Y)$ and the evaluation maps $\tilde{e v}_{k}$ to $Y$, instead of $\gamma_{k} \in A^{*}(X)$ and $e v_{k}$ (provided that $\alpha_{k}>0$ in the case of the relative invariants). We will apply the same notation in this case and just mark the cohomology classes that are pulled back from $Y$ by a tilde.

(ii) For the absolute invariants, we could use a homology class on $Y$ instead of summing over all homology classes on $Y$ that push forward to a given class on $X$. (We will never do this in this paper, however.)

The invariants obtained in this way are called the (unrestricted) Gromov-Witten invariants of $Y$, or relative Gromov-Witten invariants of $Y \subset X$, respectively.

Remark 5.2. Often the restricted invariants are really not restricted at all. As for generalization (i) in the above definition, in many cases every algebraic cohomology class in $Y$ comes from a (rational) algebraic cohomology class in $X$, notably if the dimension of $Y$ is odd (by the Lefschetz theorem) or if $X=\mathbb{P}^{N}$ and $Y$ is a generic hypersurface that is not a quadric or the cubic surface (by [S] proposition 2.1). Again by the Lefschetz theorem, (ii) is no generalization if the dimension of $Y$ is at least 3 .

Remark 5.3. If we intersect the main theorem 2.6

$$
\left(\alpha_{k} \psi_{k}+e v_{k}^{*} Y\right) \cdot\left[\bar{M}_{\alpha}(X, \beta)\right]^{\text {virt }}=\left[\bar{M}_{\alpha+e_{k}}(X, \beta)\right]^{\text {virt }}+\left[D_{\alpha, k}(X, \beta)\right]^{\text {virt }}
$$

with suitably many cotangent line classes or pull-backs from classes on $X$ or $Y$ by the evaluation maps, we obviously get many relations among the relative Gromov-Witten invariants of $Y \subset X$, the Gromov-Witten invariants of $X$ (for $\alpha=(0, \ldots, 0)$ ), and the Gromov-Witten invariants of $Y$ (as the moduli spaces of stable maps to $Y$ are included as factors in the spaces $\left.D_{\alpha, k}(X, \beta)\right)$. As for $D_{\alpha, k}(X, \beta)$ one uses the usual "diagonal trick" to express a component

$$
D_{k}(X, A, B, M)=\bar{M}_{\left|\alpha^{(0)}\right|+r}\left(Y, \beta^{(0)}\right) \times_{Y^{r}} \prod_{i=1}^{r} \bar{M}_{\alpha^{(i)} \cup\left(m^{(i)}\right)}\left(X, \beta^{(i)}\right)
$$


(and its virtual fundamental class) by the cartesian diagram

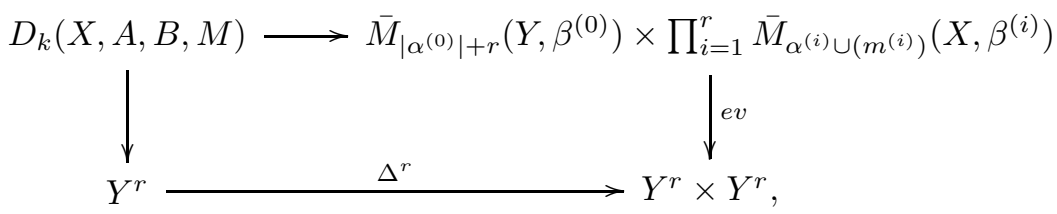

i.e. intersection products on $D_{k}(X, A, B, M)$ become intersection products of the same classes on products of moduli spaces of (absolute and relative) stable maps, with additional classes coming from the diagonal. So the term $\left[D_{\alpha, k}(X, \beta)\right]^{\text {virt }}$ in the main theorem will turn into a sum of products of Gromov-Witten invariants of $Y$ and relative Gromov-Witten invariants of $Y \subset X$.

Remark 5.4. In what follows we only want to look at the restricted (relative) Gromov-Witten invariants. It is not obvious that this is possible, as even if we only use pull-backs of classes from $X$ at the marked points $x_{1}, \ldots, x_{n}$, the classes from the diagonal trick in the terms $D_{\alpha, k}(X, \beta)$ (see above) will throw in classes from $Y$. To see that these do not do any harm we will first show in the next two lemmas that absolute as well as relative invariants vanish if they contain exactly one class from $Y$ and this class lies in the orthogonal complement $A^{*}(X)^{\perp}$ of $i^{*} A^{*}(X)$ in $A^{*}(Y)$. (These lemmas can obviously be skipped if $A^{*}(X)^{\perp}=\emptyset$, which is often the case by remark 5.2).

Lemma 5.5. Let $\tilde{\gamma}_{1} \in A^{*}(X)^{\perp}$ and $\gamma_{2}, \ldots, \gamma_{n} \in A^{*}(X)$. Then for any $\beta \in H_{2}^{+}(X)$ we have $I_{n, \beta}^{Y}\left(\tilde{\gamma}_{1} \psi^{k_{1}}, \gamma_{2} \psi^{k_{2}}, \ldots, \gamma_{n} \psi^{k_{n}}\right)=0$.

Proof. (This is a variant of proposition 4 in $[\mathrm{P}]$.) Consider the cartesian diagram (see lemma 4.2)

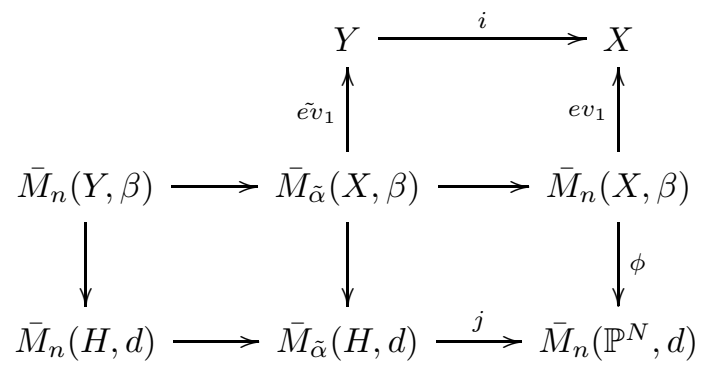

where $\tilde{\alpha}=(1,0, \ldots, 0)$. Let $\pi: \bar{M}_{n+1}\left(\mathbb{P}^{N}, d\right) \rightarrow \bar{M}_{n}\left(\mathbb{P}^{N}, d\right)$ be the universal map and $f: \bar{M}_{n+1}\left(\mathbb{P}^{N}, d\right) \rightarrow \mathbb{P}^{N}$ its evaluation map. Let $E$ be the kernel of the surjective bundle morphism $\pi_{*} f^{*} \mathcal{O}(H) \rightarrow e v_{1}^{*} \mathcal{O}(H)$ given by evaluation. By $[\mathrm{P}$ construction 2.1 and proposition 4 we have that $\left[\bar{M}_{n}(H, d)\right]=j^{*}\left(c_{t o p}(E) \cdot\left[\bar{M}_{n}\left(\mathbb{P}^{N}, d\right)\right]\right)$. Intersecting with $\left[\bar{M}_{n}(X, \beta)\right]^{\text {virt }}$ yields by lemma 4.2

$$
\left[\bar{M}_{n}(Y, \beta)\right]^{v i r t}=i^{!}\left(\phi^{*} c_{t o p}(E) \cdot\left[\bar{M}_{n}(X, \beta)\right]^{v i r t}\right)
$$


on $\bar{M}_{\tilde{\alpha}}(X, \beta)$. Moreover, the class $\gamma=\psi^{k_{1}} \cdot e v_{2}^{*} \gamma_{2} \cdot \psi^{k_{2}} \cdots e v_{n}^{*} \gamma_{n} \cdot \psi^{k_{n}}$ is actually defined on $\bar{M}_{n}(X, \beta)$. Therefore we get

$$
\begin{aligned}
I_{n, \beta}^{Y}\left(\tilde{\gamma}_{1} \psi^{k_{1}}, \gamma_{2} \psi^{k_{2}}, \ldots, \gamma_{n} \psi^{k_{n}}\right) & =\tilde{\gamma}_{1} \cdot \tilde{e v}_{1 *} i^{!}\left(\gamma \cdot \phi^{*} c_{t o p}(E) \cdot\left[\bar{M}_{n}(X, \beta)\right]^{v i r t}\right) \\
& =\tilde{\gamma}_{1} \cdot i^{*} e v_{1 *}\left(\gamma \cdot \phi^{*} c_{t o p}(E) \cdot\left[\bar{M}_{n}(X, \beta)\right]^{v i r t}\right) \\
& =0
\end{aligned}
$$

as $\tilde{\gamma}_{1} \in A^{*}(X)^{\perp}$.

Lemma 5.6. Assume that $\alpha_{1}>0$. Let $\tilde{\gamma}_{1} \in A^{*}(X)^{\perp}$ and $\gamma_{2}, \ldots, \gamma_{n} \in A^{*}(X)$. Then $I_{\alpha, \beta}\left(\tilde{\gamma}_{1} \psi^{k_{1}}, \gamma_{2} \psi^{k_{2}}, \ldots, \gamma_{n} \psi^{k_{n}}\right)=0$.

Proof. We prove the statement by induction on $d=Y \cdot \beta, n$, and $\sum \alpha$, in that order. This means: if we want to prove the statement for an invariant with certain values of $d, n$, and $\sum \alpha$, we assume that it is true for all invariants having

(i) smaller $d$, or

(ii) the same $d$ and smaller $n$, or

(iii) the same $d$, the same $n$, and smaller $\sum \alpha$.

For $\sum \alpha=1$, i.e. $\alpha=(1,0, \ldots, 0)$, the statement follows by exactly the same calculation as in the proof of lemma 5.5, just leaving out the factor $c_{t o p}(E)$. So we can assume that $\sum \alpha>1$. If $\alpha_{1}>1$ set $k=1$, otherwise choose any $k>1$ with $\alpha_{k}>0$. By the main theorem 2.6 we have

$$
\left(\left(\alpha_{k}-1\right) \psi_{k}+e v_{k}^{*} Y\right) \cdot\left[\bar{M}_{\alpha-e_{k}}(X, \beta)\right]^{v i r t}=\left[\bar{M}_{\alpha}(X, \beta)\right]^{v i r t}+\left[D_{\alpha-e_{k}, k}(X, \beta)\right]^{v i r t} .
$$

Intersect this equation with $\tilde{e} v_{1}^{*} \tilde{\gamma}_{1} \cdot \psi^{k_{1}} \cdot e v_{2}^{*} \gamma_{2} \cdot \psi^{k_{2}} \cdots e v_{n}^{*} \gamma_{n} \cdot \psi^{k_{n}}$. The first term on the right hand side is then exactly the desired invariant. We will show that all other terms vanish.

The term on the left hand side has the same $d$ and $n$, and smaller $\sum \alpha$. The invariant coming from the $\psi_{k}$-summand has exactly one class in $A^{*}(X)^{\perp}$ and hence vanishes by the induction hypothesis. The same is true for the invariant coming from the $e v_{k}^{*} Y$-term if $k>1$. If $k=1$, all classes in the invariant come from $X$, but the invariant contains the class $e v_{1}^{*} Y \cdot \tilde{e} \tilde{v}_{1}^{*} \tilde{\gamma}_{1}=\tilde{e} \tilde{v}_{1}^{*}\left(\tilde{\gamma}_{1} \cdot i^{*} Y\right)$, which is zero as $\tilde{\gamma}_{1} \in A^{*}(X)^{\perp}$. Hence the left hand side of the equation vanishes.

Now we look at the terms $D_{k}(X, A, B, M)$ on the right hand side that give products of (relative) invariants by the diagonal trick as described in remark 5.3. Note that the class of the diagonal in $Y \times Y$ is $\sum_{i} T_{i} \otimes T_{i}^{\vee}$, where $\left\{T_{i}\right\}$ is a basis of $A^{*}(Y)$. If we choose this basis such that it respects the orthogonal decomposition $A^{*}(Y)=i^{*} A^{*}(X) \oplus A^{*}(X)^{\perp}$, then $T_{i} \in A^{*}(X)^{\perp}$ if and only if $T_{i}^{\vee} \in A^{*}(X)^{\perp}$. Hence the $i$-th diagonal (where $1 \leq i \leq r$ ) will contribute one class each to the invariants for $C^{(0)}$ and $C^{(i)}$, and either both of them are in $A^{*}(X)^{\perp}$ or none of them.

For a given term $D_{k}(X, A, B, M)$, the components $C^{(i)}$ for $i>0$ all have either smaller $d$, or the same $d$ and smaller $n$ (the latter happens only if $r=1$ and $\left.\beta^{(0)}=0\right)$. Hence by induction hypothesis $(i>0)$ or lemma $5.5(i=0)$, we know for any $i \geq 0$ that the invariant for $C^{(i)}$ vanishes if it contains exactly one class from $A^{*}(X)^{\perp}$. We show that this has always to be the case for at least one $i$. Assume that this is not true. We distinguish two cases:

(i) $x_{1} \in C^{(0)}$. Then the external components $C^{(i)}$ can have at most one class from $A^{*}(X)^{\perp}$, namely the class from the diagonal. Hence by our assumption, 
they have no such class, i.e. the diagonal contributes a class from $i^{*} A^{*}(X)$ to $C^{(i)}$ and hence also to $C^{(0)}$. But then the invariant for $C^{(0)}$ has exactly one class from $A^{*}(X)^{\perp}$, namely $\tilde{\gamma}_{1}$, which is a contradiction.

(ii) $x_{1} \in C^{(i)}$ for some $i>0$. Then by our assumption, the diagonals must contribute a class from $A^{*}(X)^{\perp}$ to $C^{(i)}$, and a class from $i^{*} A^{*}(X)$ to all other $C^{(j)}$ with $j>0$. But then we have again exactly one class from $A^{*}(X)^{\perp}$ in $C^{(0)}$, namely the one from the $i$-th diagonal. This is again a contradiction.

This shows the lemma.

Corollary 5.7. Let $X$ be a smooth projective variety and $Y \subset X$ a smooth very ample hypersurface. Assume that the Gromov-Witten invariants of $X$ are known. Then there is an explicit algorithm to compute the restricted Gromov-Witten invariants of $Y$ as well as the restricted relative Gromov-Witten invariants of $Y \subset X$.

Proof. This is now straightforward. We will compute the absolute and relative invariants at the same time, and we will use recursion on the same variables as in the previous lemma.

Assume that we want to compute a relative invariant $I_{\alpha, \beta}\left(\gamma_{1} \psi^{k_{1}}, \ldots, \gamma_{n} \psi^{k_{n}}\right)$. If $\sum \alpha=0$ then this is a Gromov-Witten invariant on $X$ and therefore assumed to be known. So we can assume that $\sum \alpha>0$. On the other hand, we can also assume that $\sum \alpha \leq Y \cdot \beta=d$, as otherwise the invariant is zero anyway by definition.

Choose $k$ such that $\alpha_{k}>0$ and intersect the main theorem 2.6

$$
\left(\left(\alpha_{k}-1\right) \psi_{k}+e v_{k}^{*} Y\right) \cdot\left[\bar{M}_{\alpha-e_{k}}(X, \beta)\right]^{\text {virt }}=\left[\bar{M}_{\alpha}(X, \beta)\right]^{\text {virt }}+\left[D_{\alpha-e_{k}, k}(X, \beta)\right]^{\text {virt }}
$$

with $e v_{1}^{*} \gamma_{1} \cdot \psi^{k_{1}} \cdots e v_{n}^{*} \gamma_{n} \cdot \psi^{k_{n}}$. Then the first term on the right hand side is the invariant that we want to compute. We will show that all other terms in the equation are recursively known.

This is obvious for the invariants on the left hand side, since they have the same $d$, same $n$, and smaller $\sum \alpha$. Now look at a term coming from $D_{k}(X, A, B, M)$ on the right hand side, it is a product of invariants for the components $C^{(i)}$ for $i=0, \ldots, r$. First we will show that we only get products of restricted invariants. The invariant for the components $C^{(i)}$ for $i>0$ can have at most one class from $A^{*}(X)^{\perp}$, namely from the diagonal. But if it has exactly one it vanishes by lemma 5.6, so it has none. This means that it is a restricted invariant, and moreover that the diagonal contributes only classes from $A^{*}(X)$ to the invariant for $C^{(0)}$. This means that the invariant for $C^{(0)}$ is also a restricted one.

Now, as in the previous lemma, the invariants for the components $C^{(i)}$ for $i>0$ all have either smaller $d$, or the same $d$ and smaller $n$, and are therefore recursively known. The Gromov-Witten invariant for the component $C^{(0)}$ can certainly have no bigger $d$. We will show now that it cannot have the same $d$ either. Assume the contrary, then we must have $r=0$. But then the dimension condition says

$$
\begin{gathered}
\operatorname{vdim} \bar{M}_{\alpha}(X, \beta)=\operatorname{vdim} \bar{M}_{n}(Y, \beta) \\
\Longleftrightarrow \operatorname{vdim} \bar{M}_{n}(X, \beta)-\sum \alpha=\operatorname{vdim} \bar{M}_{n}(X, \beta)-d-1,
\end{gathered}
$$

i.e. $\sum \alpha=d+1>d$, which is a contradiction. Hence also the invariant for $C^{(0)}$ has smaller $d$. In summary, we have seen that we can compute the desired relative Gromov-Witten invariant. 
Now we compute the absolute Gromov-Witten invariants for the same values of $d$ and $n$. Assume that there is such an invariant $I_{n, \beta}^{Y}\left(\gamma_{1} \psi^{k_{1}}, \gamma_{2} \psi^{k_{2}}, \ldots, \gamma_{n} \psi^{k_{n}}\right)$. Without loss of generality we may assume that $n>0$ (if $n=0$ we can just add one marked point and require it to be on $Y$, which changes the invariant only by a factor of $d$ according to the divisor axiom). Set $\alpha=(d+1,0, \ldots, 0)$. Now consider exactly the same equation (8) as above and intersect it again with $e v_{1}^{*} \gamma_{1} \cdot \psi^{k_{1}} \cdots e v_{n}^{*} \gamma_{n} \cdot \psi^{k_{n}}$. The dimension calculation (9) above then shows that the term $\left[\bar{M}_{n}(Y, \beta)\right]^{v i r t}$ and hence the desired Gromov-Witten invariant will appear on the right hand side of our equation as one term among the $D_{k}(X, A, B, M)$. The term coming from $\bar{M}_{\alpha}(X, \beta)$ will vanish as $\sum \alpha>d$, and all other terms are known recursively by exactly the same arguments as above for the relative invariants.

Remark 5.8. Although we have just shown that all restricted Gromov-Witten invariants of $Y \subset X$ can be computed from the Gromov-Witten invariants of $X$, only a very small subset of them is needed if one is only interested in the GromovWitten invariants of $Y$. First of all, analyzing the algorithm given in the proof above, one sees that it is sufficient to consider relative invariants of the form $I_{\left(\alpha_{1}, 0, \ldots, 0\right), \beta}\left(\gamma_{1} \psi_{1}^{k_{1}}, \gamma_{2}, \ldots, \gamma_{n}\right)$, i.e. we need multiplicities and cotangent line classes at only one of the marked points. In fact, in many cases it will be sufficient to look at invariants with only one marked point - the WDVV equations of $Y$ can then be used to compute all Gromov-Witten invariants of $Y$. In a forthcoming paper we will give some explicit examples along these lines and show how corollary 5.7 can be used to reprove and generalize the "mirror symmetry" type formulas for Gromov-Witten invariants of certain hypersurfaces [B], [G], [LLY].

\section{REFERENCES}

[B] K. Behrend, Gromov-Witten invariants in algebraic geometry, Inv. Math. 127 (1997), no. 3, 601-617.

[Be] A. Bertram, Another way to enumerate rational curves with torus actions, preprint math.AG/9905159.

[BF] K. Behrend, B. Fantechi, The intrinsic normal cone, Inv. Math. 128 (1997), no. 1, 45-88.

[BM] K. Behrend, Y. Manin, Stacks of stable maps and Gromov-Witten invariants, Duke Math. J. 85 (1996), no. 1, 1-60.

[EGA4] A. Grothendieck, Éléments de géometrie algébrique IV, IHES 32 (1967).

[F] W. Fulton, Intersection theory, Springer 1984.

[G] A. Givental, Equivariant Gromov-Witten invariants, Internat. Math. Res. Notices 13 (1996), 613-663.

[IP1] E. Ionel, T. Parker, Gromov-Witten invariants of symplectic sums, Math. Res. Lett. 5 (1998), no. 5, 563-576.

[IP2] E. Ionel, T. Parker, Relative Gromov-Witten invariants, preprint math.SG/9907155.

[K] M. Kontsevich, Enumeration of rational curves via torus actions, The moduli space of curves (Texel Island 1994), Birkhäuser Progr. in Math. 129 (1995), 335-368.

[LLY] B. Lian, K. Liu, S. Yau, Mirror principle I, Asian J. of Math. 1 (1997), no. 4, 729-763.

[LR] A. Li, Y. Ruan, Symplectic surgery and Gromov-Witten invariants of Calabi-Yau 3-folds I, preprint math.AG/9803036.

[P] R. Pandharipande, Rational curves on hypersurfaces (after A. Givental), preprint math.AG/9806133.

[R] Y. Ruan, Surgery, quantum cohomology and birational geometry, preprint math.AG/ 9810039.

[S] T. Shioda, Algebraic cycles on hypersurfaces in $\mathbb{P}^{N}$, Algebraic geometry, Sendai 1985, Adv. Stud. Pure Math. 10 (1987), 717-732.

[V] R. Vakil, The enumerative geometry of rational and elliptic curves in projective space,

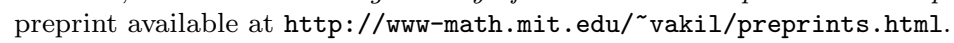


Harvard University, Department of Mathematics, Science Center, 1 Oxford Street, Cambridge, MA 02138, USA

E-mail address: andreas@math.harvard.edu 